Pacific

Journal of

Mathematics

A GEOMETRIC SPECTRAL THEORY FOR $n$-TUPLES OF SELF-ADJOINT OPERATORS IN FINITE VON NEUMANN ALGEBRAS: II

Charles A. Akemann and Joel Anderson

Volume $205 \quad$ No. 2

August 2002 


\title{
A GEOMETRIC SPECTRAL THEORY FOR $n$-TUPLES OF SELF-ADJOINT OPERATORS IN FINITE VON NEUMANN ALGEBRAS: II
}

\author{
Charles A. Akemann and Joel Anderson
}

\begin{abstract}
Given an $n$-tuple $\left\{b_{1}, \ldots, b_{n}\right\}$ of self-adjoint operators in a finite von Neumann algebra $M$ and a faithful, normal tracial state $\tau$ on $M$, we define a map $\Psi$ from $M$ to $\mathbb{R}^{n}$ by $\Psi(a)=\left(\tau(a), \tau\left(b_{1} a\right), \ldots, \tau\left(b_{n} a\right)\right)$. The image of the positive part of the unit ball under $\Psi$ is called the spectral scale of $\left\{b_{1}, \ldots, b_{n}\right\}$ relative to $\tau$ and is denoted by $B$. In a previous paper with Nik Weaver we showed that the geometry of $B$ reflects spectral data for real linear combinations of the operators $\left\{b_{1}, \ldots, b_{n}\right\}$. For example, we showed that an exposed face in $B$ is determined by a certain pair of spectral projections of a real linear combination of the $b_{i}$ 's. In the present paper we extend this study to faces that are not exposed. In order to do this we need to introduce a recursive method for describing faces of compact convex sets in $\mathbb{R}^{n}$. Using this new method, we completely describe the structure of arbitrary faces of $B$ in terms of $\left\{b_{1}, \ldots, b_{n}\right\}$ and $\tau$. We also study faces of convex, compact sets that are exposed by more than one hyperplane of support (we call these sharp faces). When such faces appear on $B$, they signal the existence of commutativity among linear combinations of the operators $\left\{b_{1}, \ldots, b_{n}\right\}$. Although many of the conclusions of this study involve too much notation to fit nicely in an abstract, there are two results that give their flavor very well. Theorem 6.1: If the set of extreme points of $B$ is countable, then $N=\left\{b_{1}, \ldots, b_{n}\right\}^{\prime \prime}$ is abelian. Corollary 5.6: $B$ has a finite number of extreme points if and only if $N$ is abelian and has finite dimension.
\end{abstract}

\section{Introduction.}

In this work we continue the investigation begun in [1] of the spectral relationships within an $n$-tuple of self-adjoint operators in a finite von Neumann algebra as reflected in the geometry of the corresponding spectral scale. Let us begin by introducing some notation and reviewing some of the results in $[\mathbf{1}]$. 
The following notation will apply throughout the rest of the paper without explicit reference.

Notation 0.1. Let $M$ denote a finite von Neumann algebra equipped with a faithful normal tracial state $\tau$, let $n$ denote a positive integer, and let $b_{1}, \ldots, b_{n}$ denote an $n$-tuple of self-adjoint operators in $M$. We define a map $\Psi$ from $M$ to $\mathbb{R}^{n+1}$ by the formula

$$
\Psi(a)=\left(\tau(a), \tau\left(b_{1} a\right), \ldots, \tau\left(b_{n} a\right)\right)
$$

and write $B=\Psi\left(M_{1}^{+}\right)$, where $M_{1}^{+}=\{a \in M: 0 \leq a \leq 1\}$. Since $\tau$ is normal and $M_{1}^{+}$is weak ${ }^{*}$ compact and convex, it follows that $B$ is a compact, convex subset of $\mathbb{R}^{n+1}$. We call $B$ the spectral scale of the $b_{i}$ 's relative to $\tau$.

We use parentheses $[()$,$] to denote vectors in \mathbb{R}^{n}$ and angle brackets $[\langle\rangle$, to denote inner products.

(1) We let $N$ denote the von Neumann subalgebra of $M$ generated by $b_{1}, \ldots, b_{n}$ and the identity.

(2) If $p$ and $q$ are projections in $M$ and $p \leq q$, then the order interval that they determine is

$$
[p, q]=\left\{a \in M_{1}^{+}: p \leq a \leq q\right\} .
$$

(3) For each nonzero vector $\mathbf{t}=\left(t_{1}, \ldots, t_{n}\right) \in \mathbb{R}^{n}$ we write

$$
b_{\mathbf{t}}=t_{1} b_{1}+\ldots+t_{n} b_{n} .
$$

(4) By a spectral pair we mean a pair of the form $(s, \mathbf{t})$, where $s$ is a real number and $\mathbf{t}$ is a nonzero vector in $\mathbb{R}^{n}$. Our standard way of embedding $\mathbb{R}^{n}$ into $\mathbb{R}^{n+1}$ is $\mathbf{t} \rightarrow(0, \mathbf{t})$.

(5) If $(s, \mathbf{t})$ is a spectral pair, then $p_{\mathbf{t}, s}^{+}$and $p_{\mathbf{t}, s}^{-}$denote the spectral projections of $b_{\mathbf{t}}$ determined by the intervals $(-\infty, s]$ and $(-\infty, s)$. We call $p_{\mathbf{t}, s}^{ \pm}$the spectral interval projections determined by $s$ and $b_{\mathbf{t}}$.

(6) If $(s, \mathbf{t})$ is a spectral pair and $\alpha$ is a real number, then $P(\mathbf{t}, s, \alpha)$ denotes the hyperplane in $\mathbb{R}^{n+1}$ defined by the formula

$$
-s x_{0}+t_{1} x_{1}+\cdots+t_{n} x_{n}=\alpha
$$

and we write $P^{\uparrow}(\mathbf{t}, s, \alpha)$ for the half-space defined by the inequality

$$
-s x_{0}+t_{1} x_{1}+\cdots+t_{n} x_{n} \geq \alpha .
$$

(7) If $0 \leq s \leq 1$, then the isotrace slice of $B$ at $s$ is by definition

$$
I_{s}=\left\{\mathbf{x}=\left(x_{0}, \ldots, x_{n}\right) \in B: x_{0}=s\right\} .
$$

The notion of an isotrace slice may seem artificial, but, as we shall show in a forthcoming paper in the International Journal of Mathematics, for $n=2$ it essentially contains the more familiar concept of the numerical range of 
the operator $c=b_{1}+i b_{2}$. Isotrace slices are particularly useful when there are two operators so that $B \subset \mathbb{R}^{3}$ as will be seen in $\S 6$ of this paper.

As shown in [1], the geometry (i.e., the facial structure) of the spectral scale $B$ contains information about the spectrum and spectral projections of real linear combinations of the $b_{1}, \ldots, b_{n}$. In this paper we complete this part of the theory by giving an exact description of an arbitrary face of $B$ in terms of the $b_{i}$ 's. In [1] this was done only for the case $n=1$, and it is useful to begin by presenting a description of these results.

Assume $n=1$ and $b_{1}=b$. Thus, $B$ is a convex, compact subset of the plane so that a face in $B$ is either an extreme point or a line segment. Also, for each real $s$ write $p_{s}^{+}$, (resp., $p_{s}^{-}$) for the spectral projection of $b$ corresponding to the interval $(-\infty, s]$ (resp., $(-\infty, s)$ ). Finally write $\sigma(b)$ for the spectrum of $b$. The results obtained in [1, Theorems 1.5 and 1.6] may be summarized as follows.

(1) $B$ lies between the lines $x=0$ and $x=1$ and has "sharp points" at $\Psi(0)=(0,0)$ and $\Psi(1)=(1, \tau(b))$. Thus, the boundary of $B$ is divided into an upper and lower boundary.

(2) The spectrum of $b$ is exactly the set of slopes of the tangent lines to the lower (or upper) boundary of $B$.

(3) A point $\mathbf{x}$ in $B$ is an extreme point on the lower boundary if and only if $\mathbf{x}$ has the form $\Psi\left(p_{s}^{ \pm}\right)$for some $s \in \sigma(b)$. Extreme points on the upper boundary have the form $\Psi\left(1-p_{\mathbf{t}, s}^{ \pm}\right)$.

(4) The line segments in the lower (or upper) boundary of $B$ are in oneto-one correspondence with the eigenvalues of $b$. If $s$ is an eigenvalue for $b$ so that $p_{s}^{-}<p_{s}^{+}$and $F$ is the line segment on the lower boundary of $B$ that it determines, then $F=\Psi\left(\left[p_{s}^{-}, p_{s}^{+}\right]\right)$.

(5) The corners in the lower (or upper) boundary of the spectral scale are in one-to-one correspondence with gaps in the spectrum of the operator $b$.

Let us now review the basic facts obtained in [1] for the higher dimensional case. Since we shall refer to these facts repeatedly, it is convenient to give them local numbers.

The following is a restatement of the results in Theorems 2.3 and 2.4 in $[\mathbf{1}]$.

Theorem 0.2. The following statements hold:

(1) If $\mathbf{x}$ is an extreme point of $B$, then there is a projection $p$ in $N$ such that

$$
\Psi(p)=\mathbf{x} \text { and } \Psi^{-1}(\mathbf{x}) \cap M_{1}^{+}=\{p\} .
$$

Further, $\Psi\left(p_{\mathbf{t}, s}^{+}\right)$and $\Psi\left(p_{\mathbf{t}, s}^{-}\right)$are extreme points of $B$ for every spectral $\operatorname{pair}(s, \mathbf{t})$. 
(2) We have $\tau\left(\left(b_{\mathbf{t}}-s 1\right) p_{\mathbf{t}, s}^{+}\right)=\tau\left(\left(b_{\mathbf{t}}-s 1\right) p_{\mathbf{t}, s}^{-}\right)$. The hyperplane $P(\mathbf{t}, s, \alpha)$ is a hyperplane of support for $B$ with $B \subset P^{\uparrow}(s, \mathbf{t}, \alpha)$ if and only if

$$
\alpha=\tau\left(\left(b_{\mathbf{t}}-s 1\right) p_{\mathbf{t}, s}^{ \pm}\right) .
$$

In this case we have $\Psi\left(p_{\mathbf{t}, s}^{ \pm}\right) \in P(\mathbf{t}, s, \alpha)$.

(3) If $\alpha=\tau\left(\left(b_{\mathbf{t}}-s 1\right) p_{\mathbf{t}, s}^{ \pm}\right)$, then $F=P(\mathbf{t}, s, \alpha) \cap B$ is a face in $B$. Moreover,

$$
\Psi^{-1}(P(\mathbf{t}, s \alpha)) \cap M_{1}^{+}=\left[p_{\mathbf{t}, s}^{-}, p_{\mathbf{t}, s}^{+}\right]
$$

and

$$
F=\Psi\left(\left[p_{\mathbf{t}, s}^{-}, p_{\mathbf{t}, s}^{+}\right]\right) .
$$

Recall that a face $F$ of a convex set $C$ is said to be an exposed face if there is a hyperplane of support $P$ for $C$ such that $F=C \cap P$. If the exposed face $F$ is a single point $\mathbf{x}$, and so necessarily an extreme point of $B$, we call $\mathbf{x}$ an exposed point. (See $\S 2$ for a more detailed review of these notions).

Although it was not specifically noted in [1], Theorem 0.2 allows the complete classification of the exposed faces in the spectral scale which we record below.

Corollary 0.3. The following statements hold:

(1) $F$ is an exposed face if and only if there is a spectral pair $(s, \mathbf{t})$ such that

$$
F=\Psi\left(\left[p_{\mathbf{t}, s}^{-}, p_{\mathbf{t}, s}^{+}\right]\right) .
$$

(2) If $\mathbf{x}$ is an extreme point in the spectral scale, then $\mathbf{x}$ is an exposed point if and only if there is a spectral pair $(s, \mathbf{t})$ such that $p_{\mathbf{t}, s}^{-}=p_{\mathbf{t}, s}^{+}$ and $\mathbf{x}=\Psi\left(p_{\mathbf{t}, s}^{ \pm}\right)$.

(3) If $F$ is a face that is not an extreme point, then $F$ is an exposed face if and only if there is a spectral pair $(s, \mathbf{t})$ such that $F=\Psi\left(\left[p_{\mathbf{t}, s}^{-}, p_{\mathbf{t}, s}^{+}\right]\right)$ as in Part (1) above and $s$ is an eigenvalue for $b_{\mathbf{t}}$. In this case if we set $r=\left(p_{\mathbf{t}, s}^{+}-p_{\mathbf{t}, s}^{-}\right)$, then $r \neq 0$ and $b_{\mathbf{t}} r=s r$.

Proof. The first conclusion follows immediately from Parts (2) and (3) of Theorem 0.2.

If $\mathbf{x}$ is an exposed point, then $\mathbf{x}=\Psi\left(p_{\mathbf{t}, s}^{ \pm}\right)$by Part (1) and this immediately implies that $\mathbf{x}=\Psi\left(p_{\mathbf{t}, s}^{ \pm}\right)$because $\tau$ faithful.

Conversely, if $(s, \mathbf{t})$ is a spectral pair such that $p_{\mathbf{t}, s}^{-}=p_{\mathbf{t}, s}^{+}$and $\mathbf{x}=\Psi\left(p_{\mathbf{t}, s}^{ \pm}\right)$, then

$$
B \cap P(\mathbf{t}, s, \alpha)=\Psi\left(p_{s}^{ \pm}\right)=\Psi\left(\left[p_{\mathbf{t}, s}^{-}, p_{\mathbf{t}, s}^{+}\right]\right)
$$

is a singleton and so this face is an exposed point. 
Finally, by Part (1) and the fact that $\Psi$ is faithful, we have that $\left(p_{\mathbf{t}, s}^{+}-\right.$ $\left.p_{\mathbf{t}, s}^{-}\right) \neq 0$ exactly when the face $F=\Psi\left(\left[p_{\mathbf{t}, s}^{-}, p_{\mathbf{t}, s}^{+}\right]\right)$has positive dimension. In this case $r=\left(p_{\mathbf{t}, s}^{+}-p_{\mathbf{t}, s}^{-}\right) \neq 0$ and we conclude that $b_{\mathbf{t}} r=s r$ from spectral theory.

The results to be presented below concern the following topics:

- A general analysis of a face of a compact, convex set in $\mathbb{R}^{n}$. ( $\S 2$ and $\S 5)$.

- A complete description of the facial structure of the spectral scale in the general case. ( $(3)$.

- An analysis of the "corners" of the spectral scale in higher dimensions. ( $(4)$.

- Applications of the results in $\S 4$ to show how geometric properties of $B$ imply the existence of central projections in $N$. ( $\S 5$ and $\S 6)$.

Let us now describe our results in more detail. Although we defined exposed faces of convex compact subsets in $\mathbb{R}^{n}$ using hyperplanes of support, there is also an equivalent formulation in terms of linear functionals. From this point of view, a face $F$ in the (convex, compact) set $C$ is said to be exposed if there is linear functional $f$ and a scalar $t$ such that $f(\mathbf{x})=t$ for each $\mathbf{x}$ in $F$ and $f(\mathbf{x})<t$ for each $\mathbf{x}$ in $C \backslash F$. In this case, we say that $f$ exposes $F$. Since the former definition is more geometric it fits better with the emphasis of this paper, but it will sometimes be convenient to use the latter, more algebraic, definition.

Observe that not every face need be exposed. For example, it is easy to construct a convex set in two dimensions that has a face in its boundary of dimension one such that the end points of this line segment (which are extreme points) are not exposed. We wish to make distinctions among exposed faces as follows. The exposed face $F$ is said to be have degree $\mathbf{k}$ if $k+1$ is the cardinality of the largest linearly independent set of linear functionals that expose $F$. In terms of hyperplanes, this means that there are $k+1$ hyperplanes of support for $F$ such that their normal vectors are linearly independent. We study such faces in $\S 4$.

In $\S 3$ we show that a general face of the spectral scale provides spectral information for linear combinations of the defining $b_{i}$ 's and certain cutdowns of these operators. If $F$ is not exposed, then a condition similar to that of Corollary 0.3 (1) holds, except that it is first necessary to cut down by a certain spectral projection. This analysis allows us to complete the characterization of the extreme points of the spectral scale which was begun in $[\mathbf{1}]$. 
Generalized "corners" are studied in $\S 4$. A corner of a convex planar set is a point on the boundary that admits more than one tangent line of support. The natural generalization of this notion in higher dimensions is a face that admits more than one hyperplane of support, i.e., a face of degree $k$ for some $1<k \leq n$. We call such faces sharp faces since they generalize sharp (i.e., non-differentiable) corners on the boundary of a 2 dimensional convex set.

Our main result on this topic can be described as follows. In two dimensions, a corner of a convex set admits precisely two tangent lines of support with linearly independent normal vectors. In higher dimensions, there can be much wider variation and a new phenomenon occurs.

Specifically, in the case of a spectral scale formed by a single operator, a tangent line of support for a face of the spectral scale is determined by real numbers $s$ and $\alpha$, where $s \in \sigma(b)$. In the general case where there are $n b_{i}$ 's, the hyperplanes of the support for a sharp face are determined by a sequence $\left(s_{1}, \mathbf{t}_{1}\right), \ldots,\left(s_{k}, \mathbf{t}_{k}\right)$ of spectral pairs and real numbers $\alpha_{1}, \ldots, \alpha_{k}$.

For example, suppose $F$ is a sharp face which is contained in hyperplanes of the form $P\left(\mathbf{t}_{1}, s_{1}, \alpha_{1}\right)$ and $P\left(\mathbf{t}_{2}, s_{2}, \alpha_{2}\right)$, where the spectral pairs $\left(s_{1}, \mathbf{t}_{1}\right)$ and $\left(s_{2}, \mathbf{t}_{2}\right)$ are linearly independent. If $\mathbf{t}_{1}=\mathbf{t}_{2}=\mathbf{t}$ and $s_{1}<s_{2}$, then, just as in two dimensions, the interval $\left(s_{1}, s_{2}\right)$ lies in a gap in the spectrum of $b_{\mathbf{t}}$. On the other hand if $\left\{\mathbf{t}_{1}, \mathbf{t}_{2}\right\}$ is a linearly independent set, then something new occurs. In this case, there is a projection $r$ that commutes with $b_{\mathbf{t}_{1}}$ and $b_{\mathbf{t}_{2}}$ and such that

$$
b_{\mathbf{t}_{1}} r=s_{1} r \text { and } b_{\mathbf{t}_{2}} r=s_{2} r .
$$

If there are $k$ hyperplanes containing $F$, with linearly independent vectors $\mathbf{t}_{1}, \ldots, \mathbf{t}_{k}$, then the projection $r$ commutes with each $b_{\mathbf{t}_{i}}$ and the compression of each $b_{\mathbf{t}_{i}}$ to $r$ is a scalar.

Next, we observe in $\S 5$ that if $k=n$ for the sharp face $F$ of the previous paragraph, then the projection $r$ commutes with each $b_{i}$ and so $r$ is a nontrivial central projection in $N$. Put colloquially, if we can "wobble" a sharp face in all $n$ "t-directions", then the center of $N$ is not trivial. $\S 6$ is devoted to showing that if $B$ has a countable number of extreme points, then $N$ is abelian.

\section{Examples.}

As noted in the introduction, one can "see" if $N$ is abelian from the shape of the spectral scale in the finite dimensional case, but the situation in infinite dimensions is much more complicated. In this section we present several examples that illustrate this point. Other examples of the spectral scale in $\mathbb{R}^{2}$ were presented in [1] at the end of $\S 1$. Examples for which $N$ is finite dimensional and noncommutative appear in a forthcoming paper. We begin with an example where $N$ is abelian and $B$ has a countable number of extreme points. 
Example 1.1. Let $b$ denote the diagonal operator on $\ell^{2}(\mathbb{N})$ defined by $b\left(r_{k}\right)=(1 / k) r_{k}, \quad k=1,2, \ldots$, where the $r_{k}$ 's denote the diagonal projections of rank one and let $M$ be the von Neumann algebra generated by $b$ and the identity (so that $M=\ell^{\infty}(\mathbb{N})$ ). Also, define a trace $\tau$ on $M$ by $\tau\left(\left\{t_{k}\right\}\right)=\sum t_{k} / 2^{k}$. Since the spectrum of $b$ is exactly $\{1 / k\}$ together with 0 , it is countable. By Theorem 1.5 of [1] the spectral scale of $\{b, \tau\}$ is a 2 dimensional, convex, compact set with upper boundary curve consisting of line segments with decreasing slopes - specifically the slopes $\{1 / k\}$. Thus the algebra $N$ is abelian (it equals $M$ ) and infinite dimensional, and the set of extreme points of $B$ is countable.

As the next example shows, even if $N$ is abelian and all linear combinations of $\left\{b_{1}, b_{2}\right\}$ have countable spectrum, $B$ may still have uncountably many extreme points.

Example 1.2. Let $M$ and $\tau$ be as in Example 1.1 and select positive real sequences $\left\{s_{k}\right\}$ and $\left\{t_{k}\right\}$, each of which converges to 0 , and such that the sequence $\left\{t_{k} / s_{k}\right\}$ takes on every rational value between 0 and infinity. Also, let $b_{1}$ and $b_{2}$ denote the corresponding diagonal operators in $\ell^{\infty}(\mathbb{N})$. Since the two sequences converge to 0 , every linear combination of $b_{1}$ and $b_{2}$ is compact. In particular, each linear combination of $b_{1}$ and $b_{2}$ has countable spectrum.

To see that $B$ has an uncountable number of extreme points, fix an irrational number $\alpha$, write $\mathbf{t}=(\alpha,-1)$ and consider the operator $b_{\mathbf{t}}=\alpha b_{1}-b_{2}$. Observe that

$$
\alpha s_{k}-t_{k}<0 \Longleftrightarrow \alpha<\frac{t_{k}}{s_{k}}
$$

Thus, if we write

$$
G_{\alpha}=\left\{k: \alpha<t_{k} / s_{k}\right\},
$$

then $p_{\mathbf{t}, 0}^{-}$is the projection onto the subspace of $H$ spanned by the corresponding standard basis vectors and we have

$$
\tau\left(p_{\mathbf{t}, 0}^{-}\right)=\sum_{k \in G_{\alpha}} \frac{1}{2^{k}} .
$$

Hence, if $\alpha \neq \alpha^{\prime}$ and we write $\mathbf{t}^{\prime}=\left(\alpha^{\prime}, 0\right)$, then $G_{\alpha} \neq G_{\alpha^{\prime}}, \tau\left(p_{\mathbf{t}, 0}^{-}\right) \neq \tau\left(p_{\mathbf{t}^{\prime}, 0}^{-}\right)$ and so

$$
p_{\mathbf{t}, 0}^{-} \neq p_{\mathbf{t}^{\prime}, 0}^{-} .
$$

Hence there are an uncountable number of spectral projections of this form and therefore by Part (1) of Theorem 0.2, the set of extreme points of $B$ is uncountable. 
As the next two examples show, it is not always "obvious" from the geometry of $\mathrm{B}$ whether or not $N$ is abelian. Finding a condition on $B$ that is necessary and sufficient for commutativity of $N$ is one of the major unsolved problems in the theory.

The pictures of the spectral scales in these examples are rather complicated. The details are much clearer in color. To view the color versions, go to

\section{http://www.math.psu.edu/anderson/pictures.}

Example 1.3. Suppose that $H=L^{2}(0,1), b_{1}$ is multiplication by $x, b_{2}$ is the projection determined by the characteristic function of the interval $[0,1 / 2]$ and the trace $\tau$ is integration. Thus, in this case $N$ is abelian. The spectral scale for these operators resembles two bed sheets, tied at their corners and billowing outwards from one another. The coinciding edges of the bed sheets form the borders of 4 two dimensional faces with parabolic boundaries that meet at the corners of the bed sheets. These faces have the same shape as the spectral scale for multiplication by $x$. (See [1, end of $\S 1]$.) Thus, the spectral scale of $b_{1}$ is visible in $B$. The spectral scale of $b_{2}$ (which is just a parallelogram) is also visible as the convex hull of the corners of the bed sheets. See Figure 1.

Example 1.4. Now let us consider a noncommutative example. Put $H=$ $L^{2}(0,1 / 2) \oplus L^{2}(1 / 2,1)$ and write

$$
b_{1}=\left[\begin{array}{cc}
a & 0 \\
0 & a+1 / 2
\end{array}\right] \text { and } b_{2}=\left[\begin{array}{cc}
1 / 2 & 1 / 2 \\
1 / 2 & 1 / 2
\end{array}\right],
$$

where $a$ is multiplication by $x$ on $[0,1 / 2]$. Thus, $b_{1}$ is still multiplication by $x$ on $[0,1]$, but the projection $b_{2}$ now does not commute with $b_{1}$. In this case the spectral scale is quite similar to the scale in the previous example, although the "billowing" of the bed sheets is more pronounced. So, based on this evidence, there does not seem to be an obvious visual method for determining when $N$ is abelian in the infinite dimensional case. See Figure 2.

\section{Preliminary results on convexity.}

How should one describe a face $F$ of a convex set $C$ in $\mathbb{R}^{n}$ ? Assuming that we have already some description of $C$, it is natural to add some conditions to that description in order to distinguish the points of $F$ from the other points of $C$. The most natural conditions, from the convexity viewpoint, should be expressed in terms of linear functionals. E.g., $F=\{x \in C: f(x)=\alpha\}$. This only works if the linear functional $f$ exposes the face $F$. Many faces are not exposed, so how can we describe them in some systematic way using linear functionals? The answer to that question is to use more than one linear functional via the recursive process described in this section. 


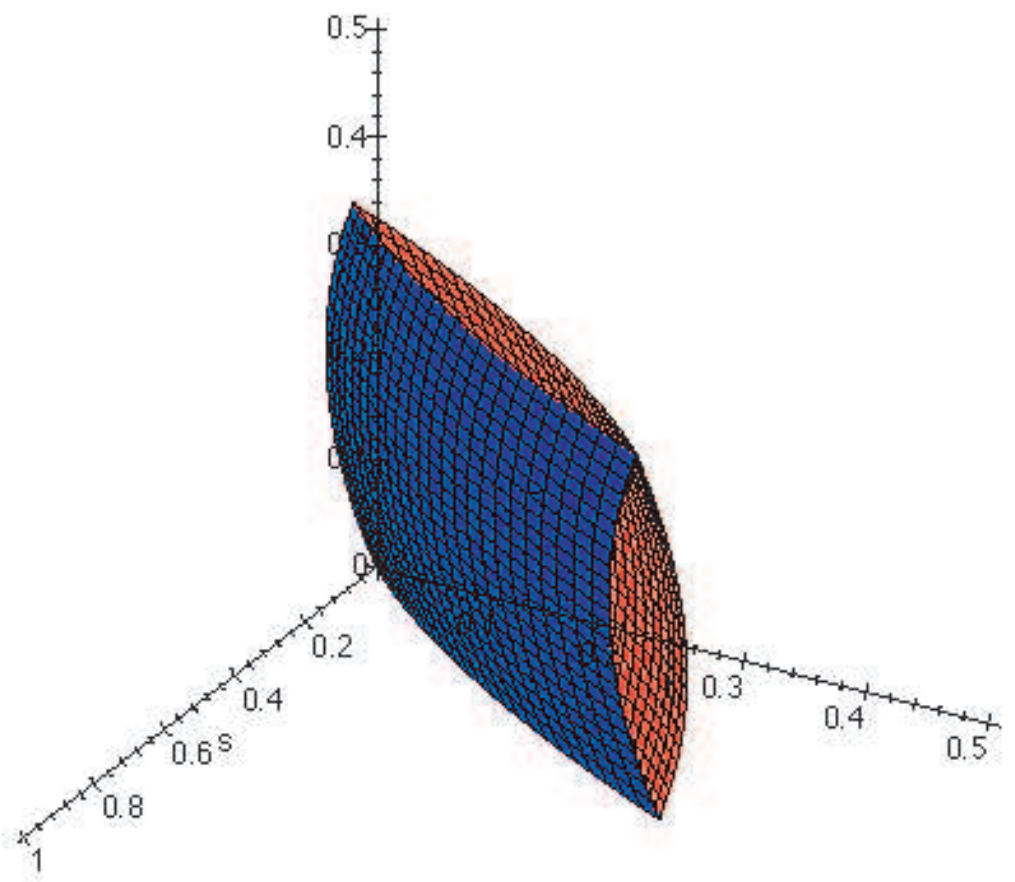

Figure 1

We begin by reviewing some of the standard notions in the theory of convexity and then present some general results that will be used in the sequel. Although some of the material that appears here does not seem to be in any of the standard texts on convexity, it is quite possible that some of the unattributed results are known.

If $\mathbf{t}=\left(t_{1}, \ldots, t_{n}\right)$ is a nonzero vector in $\mathbb{R}^{n}$ and $\alpha$ is a real number then we write

$$
P(\mathbf{t}, \alpha)=\left\{\mathbf{x}=\left(x_{1}, \ldots, x_{n}\right) \in \mathbb{R}^{n}: t_{1} x_{1}+\cdots+t_{n} x_{n}=\langle\mathbf{t}, \mathbf{x}\rangle=\alpha\right\}
$$

for the hyperplane in $\mathbb{R}^{n}$ determined by $\mathbf{t}$ and $\alpha$. The half spaces of $\mathbb{R}^{n}$ determined by the hyperplane $P(\mathbf{t}, \alpha)$ are given as follows:

$$
P^{\uparrow}(\mathbf{t}, \alpha)=\left\{\mathbf{x} \in \mathbb{R}^{n}:\langle\mathbf{t}, \mathbf{x}\rangle \geq \alpha\right\} \text {, and } P^{\downarrow}(\mathbf{t}, \alpha)=\left\{\mathbf{x} \in \mathbb{R}^{n}:\langle\mathbf{t}, \mathbf{x}\rangle \leq \alpha\right\} .
$$

Thus, $P(\mathbf{t}, \alpha)$ is the unique hyperplane in $\mathbb{R}^{n}$ passing through $\mathbf{x}$ and orthogonal to $\mathbf{t}$ and so it is natural to call the vector $\mathbf{t}$ a normal vector for this hyperplane. 


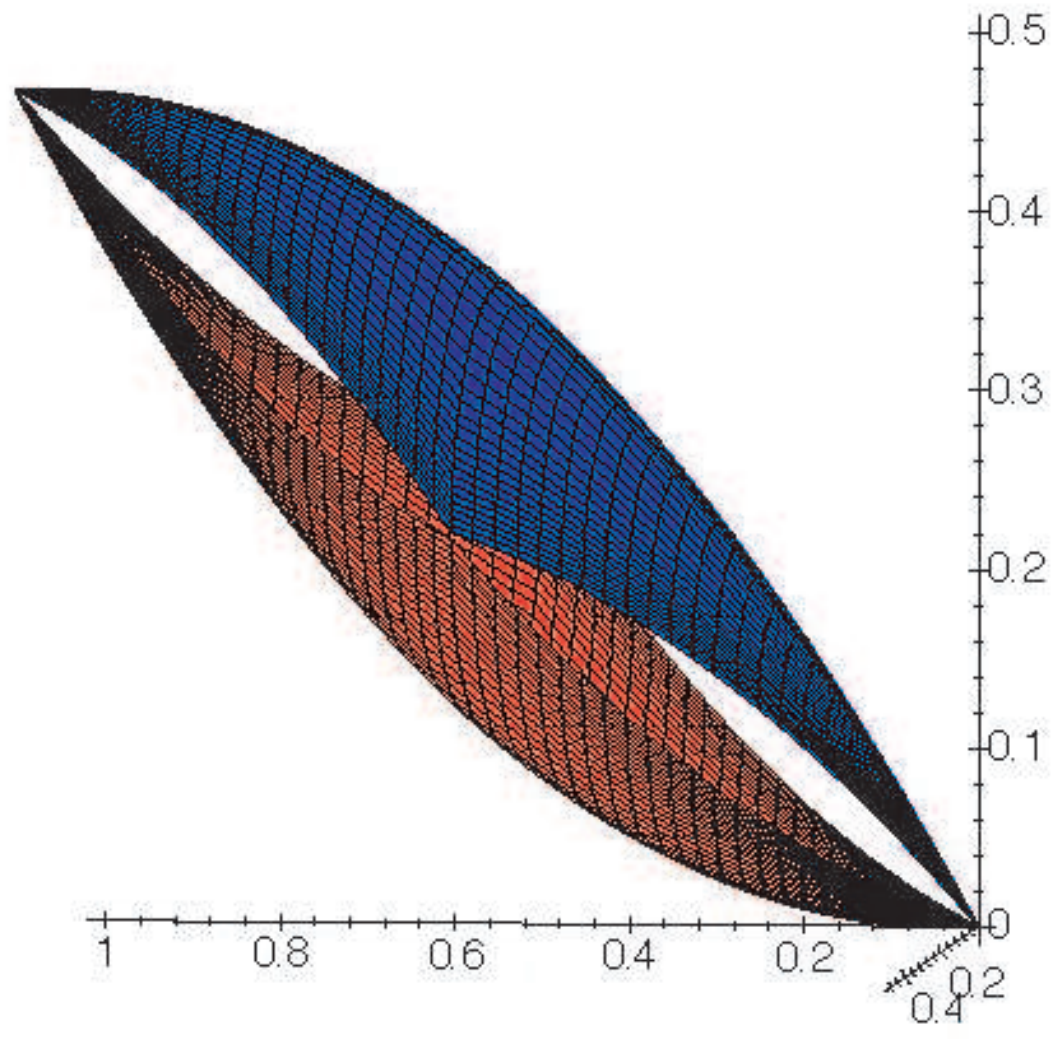

Figure 2

Now fix a convex subset $C$ of $\mathbb{R}^{n}$. The hyperplane $P(\mathbf{t}, \alpha)$ is a hyperplane of support for $C$ if there is a vector $\mathbf{x}$ in the boundary of $C$ with $\mathbf{x} \in P(\mathbf{t}, \alpha)$ and we have $(\mathbf{t}, \mathbf{x})=\alpha$ and

$$
C \subset P^{\uparrow}(\mathbf{t}, \alpha) .
$$

Note that in order for $P(\mathbf{t}, \alpha)$ to be a hyperplane of support we just as easily could have required $C \subset P^{\downarrow}(\mathbf{t}, \alpha)$. In fact this is often the definition used in many texts on convexity. We prefer to use the positive half space since it is more natural in our applications.

Note that $P(\mathbf{t}, \alpha)$ is a hyperplane of support for $C$ containing the vector $\mathbf{x} \in C$ if and only if

$$
\left\langle\mathbf{x}^{\prime}-\mathbf{x}, \mathbf{t}\right\rangle \geq 0, \quad \text { for all } \mathbf{x}^{\prime} \in C
$$


If $C$ is compact and $\mathbf{t}$ is any nonzero vector in $\mathbb{R}^{n}$, then it is straightforward to show that there is a unique real number $\alpha$ such that $P(\mathbf{t}, \alpha)$ is a hyperplane of support for $C$. Further, if $P(\mathbf{t}, \alpha)$ is a hyperplane of support for $C$, then

$$
F(\mathbf{t}, \alpha)=P(\mathbf{t}, \alpha) \cap C
$$

is a face in the boundary of $C$.

Let us now record a few general results on faces in convex sets. As above, the symbol $C$ stands for a convex set in $\mathbb{R}^{n}$. The proof of the following Theorem is essentially the same as the proof of Theorem 2.6.17 in [6], although its statement is a bit more detailed than the one given there.

Theorem 2.1. If $P\left(\mathbf{t}_{1}, \alpha_{1}\right), \ldots, P\left(\mathbf{t}_{k}, \alpha_{k}\right)$ are hyperplanes of support for $C$ such that

$$
C \cap P\left(\mathbf{t}_{1}, \alpha_{1}\right) \cap \cdots \cap P\left(\mathbf{t}_{k}, \alpha_{k}\right)=F\left(\mathbf{t}_{1}, \alpha_{1}\right) \cap \cdots \cap F\left(\mathbf{t}_{k}, \alpha_{k}\right) \neq \emptyset,
$$

$\lambda_{1}, \ldots, \lambda_{k}$ are positive real numbers and we write

$$
\begin{aligned}
\alpha_{\lambda} & =\lambda_{1} \alpha_{1}+\ldots \lambda_{k} \alpha_{k} \text { and } \\
\mathbf{t}_{\lambda} & =\lambda_{1} \mathbf{t}_{1}+\ldots \lambda_{k} \mathbf{t}_{k},
\end{aligned}
$$

then $P\left(\mathbf{t}_{\lambda}, \alpha_{\lambda}\right)$ is a hyperplane of support for $C$ and

$$
F\left(\mathbf{t}_{\lambda}, \alpha_{\lambda}\right)=P\left(\mathbf{t}_{\lambda}, \alpha_{\lambda}\right) \cap C=F\left(\mathbf{t}_{1}, \alpha_{1}\right) \cap \cdots \cap F\left(\mathbf{t}_{k}, \alpha_{k}\right) .
$$

In particular, $F\left(\mathbf{t}_{\lambda}, \alpha_{\lambda}\right)$ is an exposed face of $C$.

Proof. Fix $\mathbf{x} \in F\left(\mathbf{t}_{1}, \alpha_{1}\right) \cap \cdots \cap F\left(\mathbf{t}_{k}, \alpha_{k}\right)$. We have then that

$$
\left\langle\mathbf{t}_{i}, \mathbf{x}\right\rangle=\alpha_{i}
$$

for each $i$ and so

$$
\left\langle\mathbf{t}_{\lambda}, \mathbf{x}\right\rangle=\lambda_{1}\left\langle\mathbf{t}_{1}, \mathbf{x}\right\rangle+\cdots+\lambda_{k}\left\langle\mathbf{t}_{k}, \mathbf{x}\right\rangle=\alpha_{\lambda} .
$$

Hence $\mathbf{x} \in F\left(\mathbf{t}_{\lambda}, \alpha_{\lambda}\right)$ and therefore,

$$
F\left(\mathbf{t}_{1}, \alpha_{1}\right) \cap \cdots \cap F\left(\mathbf{t}_{k}, \alpha_{k}\right) \subset F\left(\mathbf{t}_{\lambda}, \alpha_{\lambda}\right) .
$$

Now fix $\mathbf{x} \in F\left(\mathbf{t}_{\lambda}, \alpha_{\lambda}\right)$ so that

$$
\left\langle\mathbf{x}, \mathbf{t}_{\lambda}\right\rangle=\alpha_{\lambda}=\lambda_{1} \alpha_{1}+\ldots \lambda_{k} \alpha_{k} .
$$

If we had $\mathbf{x} \notin F\left(\mathbf{t}_{i}, \alpha_{i}\right)$ for some $i$, then since $\mathbf{x} \in C$ we would have

$$
\left\langle\mathbf{t}_{i}, \mathbf{x}\right\rangle>\alpha_{i}
$$

and therefore

$$
\left\langle\mathbf{x}, \mathbf{t}_{\lambda}\right\rangle=\lambda_{1}\left\langle\mathbf{t}_{1} \mathbf{x}\right\rangle+\cdots+\lambda_{k}\left\langle\mathbf{t}_{k}, \mathbf{x}\right\rangle>\alpha_{\lambda},
$$

contradicting $(*)$. Hence,

$$
F\left(\mathbf{t}_{\lambda}, \alpha_{\lambda}\right) \subset F\left(\mathbf{t}_{1}, \alpha_{1}\right) \cap \cdots \cap F\left(\mathbf{t}_{k}, \alpha_{k}\right)
$$


and so

$$
F\left(\mathbf{t}_{\lambda}, \alpha_{\lambda}\right)=F\left(\mathbf{t}_{1}, \alpha_{1}\right) \cap \cdots \cap F\left(\mathbf{t}_{k}, \alpha_{k}\right) .
$$

Finally, since $F\left(\mathbf{t}_{1}, \alpha_{1}\right) \cap \cdots \cap F\left(\mathbf{t}_{k}, \alpha_{k}\right) \neq \emptyset$ by hypothesis, we get that $F\left(\mathbf{t}_{\lambda}, \alpha_{\lambda}\right)$ contains a point on the boundary of $C$. Further, if $\mathbf{x} \in C$, then we have

$$
\left\langle\mathbf{t}_{i}, \mathbf{x}\right\rangle \geq \alpha_{i} \quad i=1, \ldots, k
$$

and so $\left\langle\mathbf{t}_{\lambda}, \mathbf{x}\right\rangle \geq \alpha_{\lambda}$. Hence,

$$
C \subset P^{\uparrow}\left(\mathbf{t}_{\lambda}, \alpha_{\lambda}\right),
$$

$P\left(\mathbf{t}_{\lambda}, \alpha_{\lambda}\right)$ is a hyperplane of support for $C$, and $F\left(\mathbf{t}_{\lambda}, \alpha_{\lambda}\right)$ is an exposed face of $C$.

\section{Remarks 2.2.}

(1) Observe that if $\mu>0$, then $P(\mathbf{t}, \alpha)=P(\mu \mathbf{t}, \mu \alpha)$. Thus, If we let

$$
\mu=\frac{1}{\lambda_{1}+\cdots+\lambda_{k}}
$$

and replace $\mathbf{t}$ and $\alpha$ with $\mu \mathbf{t}$ and $\mu \alpha$, then we have that $\mathbf{t}_{\lambda}$ is a convex combination of the $\mathbf{t}_{i}$ 's. Hence, the proposition is equivalent to one that only considers convex combinations of vector in $\mathbb{R}^{n}$.

(2) If $F$ is a face in the boundary of $C$, then there is a hyperplane of support $P$ for $C$ that contains $F$ and such that $P$ is disjoint from the relative interior of $C$. (See [3, Theorem 11.3], for example.)

The following result is merely a restatement of Corollary 2.6.9 in [6]:

Theorem 2.3. The intersection of any family of faces of $C$ can be expressed as an intersection of $n+1$ or fewer members of the family.

Corollary 2.4. If $F$ is any nonempty face of $C$, then there is a (unique) minimal exposed face $F_{e}$ such that

$$
F \subset F_{e}
$$

Proof. Let $\mathcal{F}(F)$ denote the family of all exposed faces that contain $F$. We have that $\mathcal{F}(F) \neq \emptyset$ by remark (2) above. Write

$$
F_{e}=\bigcap_{G \in \mathcal{F}(F)} G .
$$

We have that $F_{e}$ is the intersection of a finite number of faces in $\mathcal{F}(F)$ by Theorem 2.3. Since $F \subset F_{e}$ this intersection is not empty and so $F_{e}$ is an exposed face by Theorem 2.1. It is clear from its definition that $F_{e}$ is the unique minimal exposed face containing $F$. 
Theorem 2.5. If $F$ is a face in a convex subset $C$ of $\mathbb{R}^{n}$, then there is a unique sequence

$$
F_{1} \supset \cdots \supset F_{k}
$$

such that:

(1) $F_{1}$ is the unique minimal exposed face in $C$ containing $F$.

(2) If $1<i \leq k$, then $F_{i}$ is the unique minimal exposed face of $F_{i-1}$ that contains $F$.

(3) $F=F_{k}$.

Proof. The assertions are easily established using Corollary 2.4 and a standard induction argument.

Let us call the chain $F_{1} \supset \cdots \supset F_{k}$ described above the minimal exposed facial chain determined by $F$.

\section{The facial structure of the spectral scale.}

With these preparations, we may now describe how general (i.e., nonexposed) faces of $B$ are determined by spectral properties of certain operators in $N$. It turns out that these faces are described exactly as exposed faces are except that it is necessary to work in a "cut-down" von Neumann algebra.

The key result that makes this analysis possible is the fact that a face (of positive dimension) in $B=B\left(b_{1}, \ldots, b_{n}\right)$ may be identified as a spectral scale in its own right. This scale is determined by cut-down operators of the form $r b_{1} r, \ldots, r b_{n} r$ in the cut-down von Neumann algebra $r M r$. In order to describe the situation more precisely, some new notation is required.

Suppose that $F$ is a proper face of $B$ with dimension greater than zero; i.e., $F$ is not an extreme point. In the next Lemma, it will be shown that $F$ is an affine translate of a certain related spectral scale. The new spectral scale is determined as follows. By [1, Theorems 1.1 and 2.4], we have $F=$ $\Psi\left(\left[q^{-}, q^{+}\right]\right)$where $q^{-} \leq q^{+}$are projections in $N$. Write $r=q^{+}-q^{-}$and note that since $F$ is a proper face that is not an extreme point, $0<r<1$ and therefore $0<\tau(r)<1$. Next put $M_{r}=r M r$, write

$$
\tau_{r}=\frac{1}{\tau(r)} \tau \mid M_{r}
$$

and define $\Psi_{r}$ on $M_{r}$ by the formula

$$
\Psi_{r}(x)=\left(\tau_{r}(x), \tau_{r}\left(r b_{1} r x\right), \ldots, \tau_{r}\left(r b_{n} r x\right)\right) .
$$

Finally, let $B_{F}$ denote the spectral scale determined by $\Psi_{r}$ relative to $\tau_{r}$.

Lemma 3.1. Suppose $F=\Psi\left(\left[q^{-}, q^{+}\right]\right)$is a proper face in $B$ that is not an extreme point. Using the notation developed in the preceding paragraph, the 
map $A$ defined by the formula

$$
A(\mathbf{x})=\frac{1}{\tau(r)}\left(\mathbf{x}-\Psi\left(q^{-}\right)\right)
$$

implements an affine isomorphism of $F$ onto $B_{F}$. Hence we have

$$
F=\Psi\left(q^{-}\right)+\tau(r) B_{F}=\Psi\left(q^{-}\right)+\Psi\left(\left(M_{r}\right)_{1}^{+}\right) .
$$

Proof. Observe that we have the relation

$$
\tau(r) \Psi_{r}(x)=\Psi(x), \quad x \in M_{r} .
$$

Now fix $\mathbf{x} \in F$ so that we have $\mathbf{x}=\Psi(a)$ for some $a \in\left[q^{-}, q^{+}\right]$. If we write

$$
a_{r}=\operatorname{rar} \text { so that } a=q^{-}+a_{r} \text { and } a_{r} \in\left(M_{r}\right)_{1}^{+},
$$

then we have

$$
A(\mathbf{x})=A\left(\Psi\left(q^{-}+a_{r}\right)\right)=\frac{1}{\tau(r)}\left(\Psi\left(q^{-}\right)+\Psi\left(a_{r}\right)-\Psi\left(q^{-}\right)\right)=\Psi_{r}\left(a_{r}\right),
$$

and therefore $A(\mathbf{x}) \in B_{F}$. Further, since every vector in $B_{F}$ has the form $\Psi_{r}\left(a_{r}\right)$ for some $a_{r} \in\left(M_{r}\right)_{1}^{+}$, this same calculation shows that $A$ is surjective.

Finally, suppose $\mathbf{x}_{1}$ and $\mathbf{x}_{2}$ are vectors in $F$ such that $A\left(\mathbf{x}_{1}\right)=A\left(\mathbf{x}_{2}\right)$. As $\mathbf{x}_{1}$ and $\mathbf{x}_{2}$ are in $F$, we may find operators $a_{1}$ and $a_{2}$ in $\left[q^{-}, q^{+}\right]$such that $\Psi\left(a_{i}\right)=\mathbf{x}_{i}$ and we may write

$$
a_{i}=q^{-}+a_{i, r}, \quad i=1,2,
$$

where $a_{i, r}=r a_{i} r$. With this we have

$$
A\left(\mathbf{x}_{1}\right)=\Psi_{r}\left(a_{1, r}\right)=\Psi_{r}\left(a_{2, r}\right)=A\left(\mathbf{x}_{2}\right)
$$

by $(*)$. As

$$
\mathbf{x}_{i}=\Psi\left(a_{i}\right)=\Psi\left(q^{-}+a_{i, r}\right),
$$

we get $\mathbf{x}_{1}=\mathbf{x}_{2}$ and so $A$ is injective. Hence, $A$ implements an affine isomorphism of $F$ onto $B_{F}$ and therefore

$$
F=A^{-1}\left(B_{F}\right)=\Psi\left(q^{-}\right)+\tau(r) B_{F} .
$$

The next Lemma shows that if $B$ is contained in one or more hyperplanes, then this geometric condition is equivalent to the existence of linear dependence relationships (corresponding to the hyperplanes that contain $B$ ) among $b_{1}, \ldots, b_{n}, 1$.

Lemma 3.2. If $0 \leq k<n$, and the span of $B=B\left(b_{1}, \ldots, b_{n}\right)$ has dimension $k+1$, then there are real numbers $s_{1}, \ldots, s_{n-k}$ and linearly independent vectors $\mathbf{t}_{1}, \ldots, \mathbf{t}_{n-k}$ such that $b_{\mathbf{t}_{i}}=s_{i} 1$ for $i=1, \ldots, n-k$. 
Conversely, if there are real numbers $s_{1}, \ldots, s_{n-k}$ and linearly independent vectors $\mathbf{t}_{1}, \ldots, \mathbf{t}_{n-k}$ such that $b_{\mathbf{t}_{i}}=s_{i} 1$ for $i=1, \ldots, n-k$, then the subspace spanned by $B$ has dimension at most $k+1$.

Proof. Suppose $P(\mathbf{t}, s, \beta)$ is a hyperplane in $\mathbb{R}^{n+1}$ that contains $B$ and observe that since $0 \in B$, we may take $\beta=0$. In this case, we have

$$
-s \tau(a)+\sum_{i=1}^{n} t_{i} \tau\left(b_{i} a\right)=\tau\left(\left(b_{\mathbf{t}}-s 1\right) a\right)=0
$$

for each $a \in M_{1}^{+}$. Now select $\alpha>0$ such that $0 \leq \alpha\left(b_{\mathbf{t}}-s 1\right)^{+} \leq 1$. Setting $a=\alpha\left(b_{\mathbf{t}}-s 1\right)^{+}$we get $\tau\left(\left(\alpha\left(b_{\mathbf{t}}-s 1\right)^{+}\right)^{2}\right)=0$ and since $\tau$ is faithful $\left(b_{\mathbf{t}}-s 1\right)^{+}=0$. Similarly, we get $\left(b_{\mathbf{t}}-s 1\right)^{-}=0$ and so $b_{\mathbf{t}}=s 1$.

If $B$ has dimension $k+1$, then there are $n-k$ hyperplanes of the form $P\left(\mathbf{t}_{i}, s_{i}, 0\right)$ that contain $B$ and such that their normal vectors $\left(-s_{i}, \mathbf{t}_{i}\right)$ are linearly independent. In fact we must have that the $\mathbf{t}_{i}$ 's are linearly independent. To see this, suppose that there are real numbers $\alpha_{2}, \ldots, \alpha_{n-k}$ such that

$$
\mathbf{t}_{1}=\sum_{i=2}^{n-k} \alpha_{i} \mathbf{t}_{i}
$$

In this case we get

$$
b_{\mathbf{t}_{1}}=s_{1} 1=\sum_{i=2}^{n-k} \alpha_{i} b_{\mathbf{t}_{i}}=\sum_{i=2}^{n-k} \alpha_{i} s_{i} 1 .
$$

But, then $\left(-s_{1}, \mathbf{t}_{1}\right)$ would be a linear combination of the remaining normal vectors contradicting their linear independence.

For the converse, if real scalars $\left\{s_{1}, \ldots, s_{n-k}\right\}$ and linearly independent vectors $\left\{\mathbf{t}_{1}, \ldots, \mathbf{t}_{n-k}\right\}$ exist such that $b_{\mathbf{t}_{i}}=s_{i} 1$ for all $i=1, \ldots, n-k$, then it immediately follows that every $\mathbf{x}$ in $B$ is orthogonal to each vector $\left(-s_{i}, \mathbf{t}_{i}\right)$ and so the subspace spanned by $B$ has dimension at most $k+1$.

Corollary 3.3. Using the notation developed for Lemma 3.1, if we let $N_{F}$ denote the von Neumann algebra generated by $\left\{r b_{1} r, \ldots, r b_{n} r, r\right\}$, then the following statements hold.

(1) $F$ has dimension one if and only if $N_{F}$ has dimension one.

(2) If $F$ has dimension two, then $N_{F}$ is abelian.

Proof. We have $F=\Psi\left(q^{-}\right)+\Psi\left(\left(M_{r}\right)_{1}^{+}\right)$. Suppose that $N_{F}$ has dimension one, i.e., that there are scalars $\left\{s_{1}, \ldots, s_{n}\right\}$ such that $r b_{i} r=s_{i} r$ for each $i$. In this case, if $\Psi(a) \in F$ so that $a=q^{-}+a_{r}$, where $0 \leq a_{r} \leq r$, then we 
have

$$
\begin{aligned}
\Psi(a) & =\left(\tau\left(q^{-}+a_{r}\right), \tau\left(b_{1}\left(q^{-}+a_{r}\right)\right), \ldots, \tau\left(b_{n}\left(q^{-}+a_{r}\right)\right)\right) \\
& =\Psi\left(q^{-}\right)+\left(\tau\left(a_{r}\right), \tau\left(s_{1} a_{r}\right), \ldots, \tau\left(s_{n} a_{r}\right)\right) \\
& =\Psi\left(q^{-}\right)+\tau\left(a_{r}\right)\left(1, s_{1}, \ldots, s_{n}\right) .
\end{aligned}
$$

Since $0 \leq \tau\left(a_{r}\right) \leq 1$, we get that $F$ is the line segment with end points $\Psi\left(q^{-}\right)$and $\Psi\left(q^{-}\right)+\Psi(r)$ and so $F$ has dimension one.

If $F$ has dimension one, then by Lemma $3.1 B_{F}$ has dimension one. Thus by Lemma 3.2 there are $n$ linearly independent vectors $\mathbf{t}_{1}, \ldots, \mathbf{t}_{n}$ and real numbers $s_{1}, \ldots, s_{n}$ such that $r b_{\mathbf{t}_{i}} r=s_{i} r$. Since the $\mathbf{t}_{i}$ 's are linearly independent, we get that each $r b_{i} r$ is a multiple of $r$. Hence Assertion (1) holds.

Now suppose $F$ has dimension two so that $B_{F}$ has dimension two by Lemma 3.1. In this case, by Lemma 3.2, there are $n-1$ linearly independent vectors $\mathbf{t}_{i}$ and real numbers $s_{i}$ such that $r b_{\mathbf{t}_{i}} r=s_{i} r$ for $i=1, \ldots, n-1$. This means that there is a vector $\mathbf{t}$ such that

$$
\operatorname{span}\left(r b_{\mathbf{t}} r, 1\right)=\operatorname{span}\left(r b_{1} r, \ldots, r b_{n} r, 1\right)
$$

and so $N_{F}$ is abelian.

Remark 3.4. Observe that the spectral scale described in Example 1.3 has two dimensional faces such that $M_{r}$ is abelian (in fact $M$ is abelian in this case), but $M_{r}$ is infinite dimensional since it is isomorphic to the von Neumann algebra generated by multiplication by $x$ on a suitable interval.

Let us return now to the description of all of the faces of $B$. By identifying a face $F$ with $B_{F}$, we may view all subfaces of $F$ as faces in $B_{F}$. In particular, we may apply the results in Theorem 0.2 to the exposed subfaces of $B_{F}$ to obtain data about the corresponding subfaces of $F$. This process involves a good deal of new notation and is a bit complex. We now gather this information in a definition.

Definition 3.5. In what follows, we shall consider cut-downs of operators in $M$ of the form

$$
b^{\prime}=r b r,
$$

where $r$ is a projection and $b$ is self-adjoint. Note that we may view $b^{\prime}$ as an element of $M$ or the von Neumann algebra $r M r$. If $s$ is a real number then the cut down spectral interval projections determined by $b, r$ and $s$ are the spectral projections of $b^{\prime}$ determined by the intervals $(-\infty, s)$ and $(-\infty, s]$. We compute the spectrum and the corresponding spectral projections in the algebra $M_{r}$ where the projection $r$ is the identity. 
If $\left(s_{1}, \mathbf{t}_{1}\right),\left(s_{2}, \mathbf{t}_{2}\right), \ldots,\left(s_{k}, \mathbf{t}_{k}\right)$ is a sequence of spectral pairs, then its associated facial complex is defined inductively as follows. Write

$$
r_{1}=p_{\mathbf{t}_{1}, s_{1}}^{+}-p_{\mathbf{t}_{1}, s_{1}}^{-} \text {and } b_{\mathbf{t}_{2}}^{\prime}=r_{1} b_{\mathbf{t}_{2}} r_{1} .
$$

Let $q_{\mathbf{t}_{2}, s_{2}}^{ \pm}$denote the associated cut down spectral interval projections determined by $b_{\mathbf{t}_{2}}, r_{1}$ and $s_{2}$ and write

$$
r_{2}=q_{\mathbf{t}_{2}, s_{2}}^{+}-q_{\mathbf{t}_{2}, s_{2}}^{-} \text {. }
$$

Now suppose that for some $1<i<k$ we have defined sequences

$$
\begin{aligned}
& r_{1}>r_{2}>\cdots>r_{i}, \\
& b_{\mathbf{t}_{2}}^{\prime}, \ldots, b_{\mathbf{t}_{i}}^{\prime}, \text { and } \\
& q_{\mathbf{t}_{2}, s_{2}}^{ \pm}, \ldots, q_{\mathbf{t}_{i}, s_{i}}^{ \pm}
\end{aligned}
$$

such that if $2 \leq j \leq i$, then

$$
b_{\mathbf{t}_{j}}^{\prime}=r_{j-1} b_{\mathbf{t}_{j}} r_{j-1}, \quad r_{j}=q_{\mathbf{t}_{j}, s_{j}}^{+}-q_{\mathbf{t}_{j}, s_{j}}^{-}
$$

where $q_{\mathbf{t}_{j}, s_{j}}^{ \pm}$are the cut down spectral interval projections determined by $b_{\mathbf{t}_{j}}, r_{j-1}$ and $s_{j}$.

We may then set

$$
b_{\mathbf{t}_{i+1}}^{\prime}=r_{i} b_{\mathbf{t}_{i+1}} r_{i}
$$

let $q_{\mathbf{t}_{i+1}, s_{i+1}}^{ \pm}$denote the spectral interval projections determined by $b_{\mathbf{t}_{i+1}}, r_{i}$ and $s_{i+1}$ and write

$$
r_{i+1}=q_{\mathbf{t}_{i+1}, s_{i+1}}^{+}-q_{\mathbf{t}_{i+1}, s_{i+1}}^{-} .
$$

This completes the induction.

Thus the facial complex determined by the spectral pairs $\left(s_{1}, \mathbf{t}_{1}\right), \ldots$, $\left(s_{k}, \mathbf{t}_{k}\right)$ consists of the projections $p_{\mathbf{t}_{1}, s_{1}}^{ \pm}$together with the sequences

$$
\begin{aligned}
& r_{1}>\cdots>r_{k-1}, \\
& b_{\mathbf{t}_{2}}^{\prime}, \ldots, b_{\mathbf{t}_{k}}^{\prime} \text {, and } \\
& q_{\mathbf{t}_{2}, s_{2}}^{ \pm}, \ldots, q_{\mathbf{t}_{k}, s_{k}}^{ \pm}
\end{aligned}
$$

as defined above.

Theorem 3.6. If $F$ is a face in the spectral scale and $F_{1} \supset \cdots \supset F_{k}=F$ is its associated minimal exposed facial chain, then there is a sequence $\left(s_{1}, \mathbf{t}_{1}\right),\left(s_{2}, \mathbf{t}_{2}\right), \ldots,\left(s_{k}, \mathbf{t}_{k}\right)$ of spectral pairs such that if $q_{\mathbf{t}_{2}, s_{2}}^{ \pm}, \ldots, q_{\mathbf{t}_{k}, s_{k}}^{ \pm}$denote the spectral interval projections in its associated facial complex, then $F_{1}=\Psi\left(\left[p_{\mathbf{t}_{1}, s_{1}}^{-}, p_{\mathbf{t}_{1}, s_{1}}^{+}\right]\right)$and for $2 \leq i \leq k$ we have

$$
F_{i}=\Psi\left(\left[q_{i}^{-}, q_{i}^{+}\right]\right)=\Psi\left(q_{\mathbf{t}_{i-1}, s_{i-1}}^{-}\right)+\Psi\left(\left[q_{\mathbf{t}_{i}, s_{i}}^{-}, q_{\mathbf{t}_{i}, s_{i}}^{+}\right]\right)
$$


where

$$
\begin{aligned}
& q_{i}^{-}=p_{\mathbf{t}_{1}, s_{1}}^{-}+q_{\mathbf{t}_{2}, s_{2}}^{-}+\cdots+q_{\mathbf{t}_{i}, s_{i}}^{-} \quad \text { and } \\
& q_{i}^{+}=p_{\mathbf{t}_{1}, s_{1}}^{-}+q_{\mathbf{t}_{2}, s_{2}}^{-}+\cdots+q_{\mathbf{t}_{i-1}, s_{i-1}}^{-}+q_{\mathbf{t}_{i}, s_{i}}^{+} .
\end{aligned}
$$

Proof. Let us proceed by induction. As $F_{1}$ is an exposed face in $B$, there is a spectral pair $\left(s_{1}, \mathbf{t}_{1}\right)$ such that

$$
F_{1}=\Psi\left(\left[p_{\mathbf{t}_{1}, s_{1}}^{-}, p_{\mathbf{t}_{1}, s_{1}}^{+}\right]\right) .
$$

If $F=F_{1}$, we are done.

Otherwise, write $r_{1}=p_{\mathbf{t}_{1}, s_{1}}^{+}-p_{\mathbf{t}_{1}, s_{1}}^{-}$and let $A_{1}$ denote the affine map defined in Lemma 3.1 for the face $F_{1}$ of $B$. We have that $A_{1}\left(F_{2}\right)$ is an exposed face in $B_{F_{1}}$ and so by Theorem 0.2 , there is a spectral pair $\left(s_{2}, \mathbf{t}_{2}\right)$ such that if $b_{\mathbf{t}_{2}}^{\prime}=r_{1} b_{\mathbf{t}_{2}} r_{1}$ and $q_{\mathbf{t}_{2}, s_{2}}^{ \pm}$denote the associated cut down spectral interval projections, then we have

$$
A_{1}\left(F_{2}\right)=\Psi_{r_{1}}\left(\left[q_{\mathbf{t}_{2}, s_{2}}^{-}, q_{\mathbf{t}_{2}, s_{2}}^{+}\right]\right) .
$$

Hence, by Lemma 3.1 we have

$$
F_{2}=A_{1}^{-1}\left(\Psi_{r_{1}}\left(\left[q_{\mathbf{t}_{2}, s_{2}}^{-}, q_{\mathbf{t}_{2}, s_{2}}^{+}\right]\right)\right)=\Psi\left(p_{\mathbf{t}_{1}, s_{1}}^{-}\right)+\Psi\left(\left(M_{r_{1}}\right)_{1}^{+}\right) .
$$

If $k=2$, then we are done. Otherwise, suppose that spectral pairs $\left(s_{1}, \mathbf{t}_{1}\right),\left(s_{2}, \mathbf{t}_{2}\right), \ldots,\left(s_{i}, \mathbf{t}_{i}\right)$ and sequences

$$
\begin{aligned}
& r_{1}>\cdots>r_{i-1}, \\
& b_{\mathbf{t}_{2}}^{\prime}, \ldots, b_{\mathbf{t}_{i}}^{\prime}, \text { and } \\
& q_{\mathbf{t}_{2}, s_{2}}^{ \pm}, \ldots, q_{\mathbf{t}_{i}, s_{i}}^{ \pm}
\end{aligned}
$$

have been determined as above such that if $2 \leq j<i$ and

$$
\begin{aligned}
& q_{j}^{-}=p_{\mathbf{t}_{1}, s_{1}}^{-}+q_{\mathbf{t}_{2}, s_{2}}^{-}+\cdots+q_{\mathbf{t}_{j}, s_{j}}^{-} \quad \text { and } \\
& q_{i}^{+}=p_{\mathbf{t}_{1}, s_{1}}^{-}+q_{\mathbf{t}_{2}, s_{2}}^{-}+\cdots+q_{\mathbf{t}_{j-1}, s_{j-1}}^{-}+q_{\mathbf{t}_{j}, s_{j}}^{+},
\end{aligned}
$$

then we have

$$
F_{j}=\Psi\left(\left[q_{j}^{-}, q_{j}^{+}\right]\right)=\Psi\left(q_{\mathbf{t}_{j-1}, s_{j-1}}^{-}\right)+\Psi\left(\left[q_{\mathbf{t}_{j}, s_{j}}^{-}, q_{\mathbf{t}_{j}, s_{j}}^{+}\right]\right) .
$$

With this, we may write $r_{i}=q_{\mathbf{t}_{i}, s_{i}}^{+}-q_{\mathbf{t}_{i}, s_{i}}^{-}, b_{\mathbf{t}_{i+1}}^{\prime}=r_{i} b_{\mathbf{t}_{i+1}} r_{i}$ and let $q_{\mathbf{t}_{i+1}, s_{i+1}}^{ \pm}$ denote the associated spectral interval projections. As $F_{i}$ is exposed in $F_{i-1}$, we may apply Lemma 3.1 to get the affine map $A_{i-1}$ such that $A_{i-1}\left(F_{i-1}\right)=$ $B_{F_{i-1}}$ and $A_{i}\left(F_{i}\right)$ is an exposed face in $B_{F_{i-1}}$. Hence, applying Theorem 0.2 we get a spectral pair $\left(s_{i}, \mathbf{t}_{i}\right)$ such that if we write $b_{\mathbf{t}_{i}}^{\prime}=r_{-1} b_{\mathbf{t}_{i}} r_{i-1}$ and let $q_{\mathbf{t}_{i}, s_{i}}^{ \pm}$denote the associated spectral interval projections, then we have

$$
A_{i-1}\left(F_{i}\right)=\Psi_{r_{i-1}}\left(\left[q_{\mathbf{t}_{i}, s_{i}}^{-}, q_{\mathbf{t}_{i}, s_{i}}^{+}\right]\right) .
$$

Applying $A_{i-1}^{-1}$ to $A_{i-1}\left(F_{i}\right)$, yields the desired result. 


\section{Corners and their generalizations.}

In the case of a single operator the corners on the boundary of the spectral scale are in one-to-one correspondence with the gaps in the spectrum of $b$. In this section we study analogous faces in higher dimensions.

Definition 4.1. If $C$ is a convex set in a vector space and $F$ is a face in $C$, then we say $F$ is a sharp face if it is contained in two or more hyperplanes of support.

Recall that if $A$ is an affine space in $\mathbb{R}^{n}$, then the dimension of $A$ is the dimension of $A-\mathbf{x}$, where $\mathbf{x}$ is any vector in $A$. The dimension of the convex set $C$ is by definition the dimension of the affine space that $C$ generates. The dimension is denoted by $\operatorname{dim}(C)$.

Now fix a nonempty face $F$ of $C$. Recall that a vector $\mathbf{t} \in \mathbb{R}^{n}$ is a normal vector for $F$ if $P(\mathbf{t}, \alpha(\mathbf{t}))$ is a hyperplane of support for $C$ and $C \subset P^{\uparrow}(\mathbf{t}, \alpha(\mathbf{t}))$. The normal cone of $F$ is by definition

$$
K_{F}=\left\{\mathbf{t} \in \mathbb{R}^{n}: \mathbf{t} \text { is a normal vector for } F\right\} .
$$

The following result is basic to our analysis of sharp faces in the spectral scale.

Theorem 4.2. If $F=\Psi\left(\left[q^{-}, q^{+}\right]\right)$is a face in the spectral scale $B$ and $(s, \mathbf{t})$ is a spectral pair, then the following statements are equivalent:

(1) The vector $(-s, \mathbf{t})$ is in $K_{F}$.

(2) We have $p_{\mathbf{t}, s}^{-} \leq q^{-} \leq q^{+} \leq p_{\mathbf{t}, s}^{+}$.

(3) If we write

$$
r_{1}=q^{-}, r_{2}=q^{+}-q^{-} \text {and } r_{3}=1-q^{+},
$$

set $b_{\mathbf{t}, 1}=b_{\mathbf{t}} r_{1}$ and $b_{\mathbf{t}, 2}=b_{\mathbf{t}} r_{3}$, then

$$
\begin{aligned}
b_{\mathbf{t}, 1} & \leq s r_{1}, \\
b_{\mathbf{t}} r_{2} & =s r_{2}, \\
b_{\mathbf{t}, 2} & \geq s r_{3},
\end{aligned}
$$

and $q^{ \pm} b_{\mathbf{t}}=b_{\mathbf{t}} q^{ \pm}$

In other words, the matrix for $b_{\mathbf{t}}$ determined by the projections $r_{1}, r_{2}$ and $r_{3}$ has the form

$$
b_{\mathbf{t}}=\left[\begin{array}{ccc}
b_{\mathbf{t}, 1} & 0 & 0 \\
0 & s & 0 \\
0 & 0 & b_{\mathbf{t}, 2}
\end{array}\right]
$$

In particular, the $r_{i}$ 's commute with $b_{\mathbf{t}}$. 
Proof. Suppose (1) holds and $P(\mathbf{t}, s, \alpha)$ is the hyperplane of support that contains $F$. Since $F=\Psi\left(\left[q^{-}, q^{+}\right]\right) \subset \Psi\left(\left[p_{\mathbf{t}, s}^{-}, p_{\mathbf{t}, s}^{+}\right]\right)$we get

$$
p_{\mathbf{t}, s}^{-} \leq q^{-} \leq q^{+} \leq p_{\mathbf{t}, s}^{+}
$$

by Parts (2) and (3) of Theorem 0.2. Hence (1) $\Longrightarrow(2)$. It is also clear from the above that $(2) \Longrightarrow(1)$.

Now suppose (2) holds. In this case since, $p_{\mathbf{t}, s}^{ \pm}$are the spectral interval projections for $b_{\mathbf{t}}$ and $p_{\mathbf{t}, s}^{-} \leq q^{-} \leq q^{+} \leq p_{\mathbf{t}, s}^{+}$we get

$$
b_{\mathbf{t}} q^{-} \leq s q^{-},\left(q^{+}-q^{-}\right) b_{\mathbf{t}}=s\left(q^{+}-q^{-}\right) \text {and } b_{\mathbf{t}}\left(1-q^{+}\right) \geq s\left(1-q^{+}\right) .
$$

Further we have $q^{-} b_{\mathbf{t}}=b_{\mathbf{t}} q^{-}$and $\left(1-q^{+}\right) b_{\mathbf{t}}=b_{\mathbf{t}}\left(1-q^{+}\right)$and so $b_{\mathbf{t}}$ has the desired form. Thus, $(2) \Longrightarrow(3)$.

Now suppose (3) holds. As $b_{\mathbf{t}, 1} \leq s r_{1}, b_{\mathbf{t}} r_{2}=s r_{2}$ and $b_{\mathbf{t}, 2} \geq s r_{3}$, we get

$$
p_{\mathbf{t}, s}^{-} \leq q^{-}<q^{+}<p_{\mathbf{t}, s}^{+}
$$

and so $(3) \Longrightarrow(2)$.

Theorem 4.3. If $F=\Psi\left(\left[q^{-}, q^{+}\right]\right)$is a face in the spectral scale, then $F$ is a sharp face if and only if there are linearly independent spectral pairs $\left(s_{1}, \mathbf{t}_{1}\right)$ and $\left(s_{2}, \mathbf{t}_{2}\right)$ such that

$$
F \subset P\left(\mathbf{t}_{1}, s_{1}, \alpha_{1}\right) \cap P\left(\mathbf{t}_{2}, s_{2}, \alpha_{2}\right) .
$$

In this case if we write

$$
r_{1}=q^{-}, \quad r_{2}=q^{+}-q^{-}, \text {and } r_{3}=1-q^{+},
$$

then we have:

(1) $r_{1}+r_{2}+r_{3}=1$,

(2) $b_{\mathbf{t}_{i}} r_{1} \leq s_{i} r_{1}$

(3) $b_{\mathbf{t}_{i}} r_{2}=s_{i} r_{2}$, and

(4) $b_{\mathbf{t}_{i}} r_{3} \geq s_{i} r_{3}$

for $i=1,2$ and the $r_{i}$ 's commute with $b_{\mathbf{t}}$.

Proof. The assertions are easily established using the definition of a sharp face and Theorem 4.2.

Theorem 4.4. If $F=\Psi\left(\left[q^{-}, q^{+}\right]\right)$is a proper face of $B$ and $F_{e}$ is the minimal exposed face containing $F$, so that $F_{e}=\Psi\left(\left[p_{F}^{-}, p_{F}^{+}\right]\right)$, where $p_{F}^{ \pm}$are spectral interval projections of a linear combination of the $b_{i}$ 's, then we have

$$
p_{F}^{-} \leq q^{-} \leq q^{+} \leq p_{F}^{+} .
$$

Further, if $(s, \mathbf{t})$ is a spectral pair such that $\Psi\left(\left[p_{\mathbf{t}, s}^{-}, p_{\mathbf{t}, s}^{+}\right]\right)$is an exposed face of $B$ that contains $F_{e}$, then

$$
p_{\mathbf{t}, s}^{-} \leq p_{F}^{-} \leq q^{-} \leq q^{+} \leq p_{F}^{+} \leq p_{\mathbf{t}, s}^{+} .
$$


Proof. All of the assertions are simple consequences of Parts (1) and (2) of Theorem 4.2.

Corollary 4.5. Using the notation of Theorem 4.4, a face $F=\Psi\left(\left[q^{-}, q^{+}\right]\right)$ of $B$ is an extreme point of $B$ if and only if $q^{-}=q^{+}$. In this case we have

$$
b_{\mathbf{t}}=\left[\begin{array}{cc}
b_{\mathbf{t}, 1} & 0 \\
0 & b_{\mathbf{t}, 2}
\end{array}\right],
$$

where $b_{\mathbf{t}, 1} \leq s q^{-}$and $b_{\mathbf{t}, 2} \geq s\left(1-q^{+}\right)$. The face $F=\Psi\left(q^{ \pm}\right)$is an exposed point if and only if $p_{F^{-}}=p_{F^{+}}$.

Proof. We have that $F$ is a singleton if and only if $q^{-}=q^{+}$. In this case, in the notation of Theorem 4.2, we get $r_{2}=0$ and so $b_{\mathbf{t}}$ has the desired matrix decomposition by Part (3) of Theorem 4.2. We have that $\mathbf{x}$ is an exposed point if and only if $F=F_{e}$, where $F=\{\mathbf{x}\}$. Since $F_{e}=\Psi\left(\left[p_{F}^{-}, p_{F}^{+}\right]\right)$by Theorem 4.4, the final assertion follows.

Before presenting the next result, it is convenient to introduce some new notation. Suppose $F$ is a face in a convex subset of $\mathbb{R}^{n}$. The degree of $F$ is denoted by $\operatorname{deg}(F)$ and is by definition the dimension of the normal cone $K_{F}$. Recall that, the subspace spanned by $K_{F}$ is $K_{F}-K_{F}$ and so the degree of $F$ is just the dimension of $K_{F}-K_{F}$.

Theorem 4.6. The following assertions hold:

(1) The degree of $F$ is equal to the maximal number of linearly independent vectors in $K_{F}$.

(2) We have $\operatorname{deg}(F)+\operatorname{dim}(F) \leq n$.

(3) $F$ is a sharp face if and only if $\operatorname{deg}(F)>1$.

Proof. For (1), it is clear that there can be no more than $\operatorname{deg}(F)$ such vectors. Now suppose $\left\{\mathbf{t}_{1}^{+}, \ldots, \mathbf{t}_{k}^{+}\right\}$is a maximal set of linearly independent vectors in $K_{F}$ and fix any vector $\mathbf{t}$ in $K_{F}-K_{F}$ We must have $\mathbf{t}=\mathbf{t}^{+}-\mathbf{t}^{-}$, where $\mathbf{t}^{ \pm} \in$ $K_{F}$. This means that both $\mathbf{t}^{+}$and $\mathbf{t}^{-}$are linear combinations of $\left\{\mathbf{t}_{1}^{+}, \ldots, \mathbf{t}_{k}^{+}\right\}$ and so $\mathbf{t}$ is a linear combination of $\left\{\mathbf{t}_{1}^{+}, \ldots, \mathbf{t}_{k}^{+}\right\}$. Thus, $\left\{\mathbf{t}_{1}^{+}, \ldots, \mathbf{t}_{k}^{+}\right\}$is a basis for $K_{F}-K_{F}$.

Next, since each vector in $K_{F}$ is orthogonal to the difference of any two vectors in $F$, Assertion (2) is clear. Finally, Assertion (3) follows immediately from the definitions of a sharp face and the degree of $F$.

Corollary 4.7. If $B \subset \mathbb{R}^{n+1}, F=\Psi\left(\left[q^{-}, q^{+}\right]\right)$is a face in $B$ and there are spectral pairs $\left(s_{1}, \mathbf{t}_{1}\right), \ldots,\left(s_{n}, \mathbf{t}_{n}\right)$ such that:

(1) $F \subset \Psi\left(\left[p_{\mathbf{t}_{i}, s_{i}}^{-}, p_{\mathbf{t}_{i}, s_{i}}^{+}\right]\right)$for $i=1, \ldots, n$ and

(2) $\mathbf{t}_{1}, \ldots, \mathbf{t}_{n}$ are linearly independent,

then $q^{-}$and $q^{+}$are central projections in the von Neumann algebra generated by $\left\{b_{1}, \ldots, b_{n}, 1\right\}$. 
Proof. Applying Theorem 4.3, we get that $q^{-}$and $q^{+}$commute with each $b_{\mathbf{t}_{i}}$. Since $\mathbf{t}_{1}, \ldots, \mathbf{t}_{n}$ are linearly independent, each $b_{i}$ is a linear combination of the $b_{\mathbf{t}_{i}}$ 's and so $q_{1}$ and $q^{+}$commute with each $b_{i}$.

Remark 4.8. In the notation of the last Corollary, $\operatorname{deg}(F) \geq n$, so that by Part (2) of Theorem 4.6, we get that $\operatorname{dim}(F) \leq 1$. Thus, in this case, $F$ is either a sharp extreme point or a sharp line segment in $B$.

Corollary 4.9. If $F=\Psi\left(\left[q^{-}, q^{+}\right]\right)$is a face in the spectral scale and $\left(-s_{1}, \mathbf{t}\right)$ and $\left(-s_{2}, \mathbf{t}\right)$ are distinct vectors in $K_{F}$ with $s_{1}<s_{2}$, then:

(1) The interval $\left(s_{1}, s_{2}\right)$ lies in a gap in the spectrum of $b_{\mathbf{t}}$ and

(2) $F$ is an exposed point.

Proof. Applying Theorem 4.3 (and using its notation) we get that if $r_{2}=$ $q^{+}-q^{-}$, then

$$
b_{\mathbf{t}} r_{2}=s_{1} r_{2}=s_{2} r_{2}
$$

and so $r_{2}=0$ and $q^{-}=q^{+}=q$.

Next as $\left(-s_{i}, \mathbf{t}\right) \in K_{F}$ for $i=1,2$, we get

$$
p_{\mathbf{t}_{i}, s_{i}}^{-} \leq q \leq p_{\mathbf{t}_{i}, s_{i}}^{+}
$$

for $i=1,2$. On the other hand, since $s_{1}<s_{2}$ we have $p_{\mathbf{t}_{1}, s_{1}}^{+} \leq p_{\mathbf{t}_{2}, s_{2}}^{-}$so that

$$
p_{\mathbf{t}_{2}, s_{2}}^{-} \leq q \leq p_{\mathbf{t}_{1}, s_{1}}^{+} \leq p_{\mathbf{t}_{2}, s_{2}}^{-}
$$

and therefore

$$
q=p_{\mathbf{t}_{1}, s_{1}}^{+}=p_{\mathbf{t}_{2}, s_{2}}^{-} .
$$

Hence there must be a gap in the spectrum of $b_{\mathbf{t}}$ that contains the interval $\left(s_{1}, s_{2}\right)$.

Next, if we select any $s$ such that $s_{1}<s<s_{2}$, then we have $p_{\mathbf{t}, s}^{-}=p_{\mathbf{t}, s}^{+}=q$ and so $F=\Psi\left(p_{\mathbf{t}, s}^{ \pm}\right)$. Thus, $F$ is an exposed point by Corollary 0.3 .

\section{Isolated extreme points and central projections.}

We say that an element $\mathbf{x}$ in a convex set $C$ is an isolated extreme point of $C$ if it is an extreme point and it is isolated in the set $\operatorname{ext}(C)$ of all extreme points of $C$. Our next goal is to show that isolated extreme points in $B$ are images of central projections in $N$ and further that this condition characterizes isolated extreme points in the finite dimensional case. It is convenient to begin by presenting three Lemmas. The first Lemma simply records an easy fact about convex subsets in $\mathbb{R}^{n}$. The (slick) proof of the second is due to Robert Phelps (private communication).

Recall that if $A_{1}$ and $A_{2}$ are disjoint, compact, convex subsets in a real topological vector space, then there is a continuous linear functional $f$ that 
separates $A_{1}$ and $A_{2}$ in the sense that there are real numbers $s<t$ such that

$$
f\left(\mathbf{x}_{1}\right)<s<t<f\left(\mathbf{x}_{2}\right)
$$

for all $\mathbf{x}_{1} \in A_{1}$ and $\mathbf{x}_{2} \in A_{2}[4$, Theorem $3.4(\mathrm{~b})]$. In this case we say that $s$ and $t$ are separation constants for $f$.

Lemma 5.1. If $C_{0}$ is a compact, convex subset of $\mathbb{R}^{n}, \mathbf{x}$ is a point in $\mathbb{R}^{n}$ disjoint from $C_{0}$ and $C=\operatorname{conv}\left(C_{0}, \mathbf{x}\right)$, then a linear functional $f$ exposes $\mathbf{x}$ in $C$ if and only if $f$ separates $C_{0}$ and $\{\mathbf{x}\}$.

Proof. Suppose that $f$ exposes $\mathbf{x}$ in $C$ so that there is a number $t$ such that $f(\mathbf{x})=t$ and $f(\mathbf{z})<t$ if $\mathbf{z} \in C$ and $\mathbf{z} \neq \mathbf{x}$. Write

$$
r=\sup \left\{f(\mathbf{y}): \mathbf{y} \in C_{0}\right\}
$$

and suppose $\mathbf{y}_{n}$ is a sequence in $C_{0}$ such that $\lim _{n} f\left(\mathbf{y}_{n}\right)=r$. As $C_{0}$ is compact, we may assume that $\mathbf{y}_{n}$ converges to a vector $\mathbf{y} \in C_{0}$. Since $f(\mathbf{y})=r, f$ exposes $\mathbf{x}$ and $\mathbf{y} \neq \mathbf{x}$, we must have that $r<t$. Hence, we get that $f$ separates $C_{0}$ and $\mathbf{x}$.

Now suppose that $f$ separates $C_{0}$ and $\mathbf{x}$ with separation constants $s<t$ and fix $\mathbf{z} \neq \mathbf{x} \in C$. Since $C=\operatorname{conv}\left(C_{0}, \mathbf{x}\right)$, there is a number $r$ with $0 \leq r<1$ and a vector $\mathbf{y} \in C_{0}$, such that $\mathbf{z}=r \mathbf{x}+(1-r) \mathbf{y}$. Hence, $f(\mathbf{z})<r t+(1-r) s<t$ and so $f$ exposes $\mathbf{x}$.

Lemma 5.2. If $C$ is a convex, compact subset in $\mathbb{R}^{n}$ and $\mathbf{x}$ is an isolated extreme point in $C$, then the set of linear functionals that expose $\mathbf{x}$ is a nonempty open set. Further, there are linearly independent vectors $\mathbf{t}_{1}, \ldots$, $\mathbf{t}_{n}$ and real numbers $\alpha_{1}, \ldots, \alpha_{n}$ such that the hyperplanes $P\left(\mathbf{t}_{1}, \alpha_{1}\right), \ldots$, $P\left(\mathbf{t}_{n}, \alpha_{n}\right)$ are hyperplanes of support for $C$ and

$$
P\left(\mathbf{t}_{i}, \alpha_{i}\right) \cap C=\{\mathbf{x}\}, \quad i=1, \ldots, n .
$$

Proof. Write $A$ for the closure of $\operatorname{ext}(C) \backslash\{\mathbf{x}\}$ and observe that since $\mathbf{x}$ is an isolated point in $\operatorname{ext}(C), \mathbf{x} \notin A$. Further, if $C_{0}$ denotes the closed convex hull of $A$, then $\mathbf{x} \notin C_{0}$. Indeed, if we had $\mathbf{x} \in C_{0}$, then $\mathbf{x}$ would be an extreme point of $C_{0}$. But in this case we would get that $\mathbf{x} \in A$ by Choquet's "converse" to the Krein-Milman theorem [2, Appendix B14].

As noted in the paragraph before Lemma 5.1, there is a continuous linear functional $f$ that separates $C_{0}$ and $\mathbf{x}$ and so, by Lemma $5.1 f$ exposes $\mathbf{x}$ in $C$. Hence the set of linear functionals that expose $\mathbf{x}$ is not empty.

Now suppose that $f$ is a linear functional that exposes $\mathbf{x}$. Applying Lemma 5.1, we get that there are real numbers $s<t$ such that $f$ separates $C_{0}$ and $\mathbf{x}$ with separation constants $s$ and $t$. Now select $\epsilon>0$ so that if

$$
M=\sup \left\{\|\mathbf{y}\|: \mathbf{y} \in C_{0}\right\}, \text { then } \max \{\epsilon\|\mathbf{x}\|, \epsilon M\}<\frac{t-s}{3}
$$


and let $g$ denote a linear functional with $\|f-g\|<\epsilon$. With this we get

$$
g(\mathbf{x})=f(\mathbf{x})+g(\mathbf{x})-f(\mathbf{x})>t-\epsilon\|\mathbf{x}\| \geq t-\frac{t-s}{3} .
$$

If $\mathbf{y} \in C_{0}$, then we have

$$
g(\mathbf{y})=f(\mathbf{y})+g(\mathbf{y})-f(\mathbf{y})<s+\epsilon M \leq s+\frac{t-s}{3}
$$

and so $f$ separates $C_{0}$ and $\mathrm{x}$ with separation constants $s+(t-s) / 3$ and $t-(t-s) / 3$. Applying Lemma 5.1 we get that $g$ also exposes $\mathbf{x}$ and so the set of all such linear functionals is open.

To get the final conclusion, observe that since the set of linear functionals that expose $\mathbf{x}$ is a nonempty open set, it contains a linearly independent set of $n$ linear functionals, $f_{1}, \ldots, f_{n}$. Thus there are linearly independent vectors $\mathbf{t}_{1}, \ldots, \mathbf{t}_{n}$ such that $f_{i}$ is given by the inner product with $\mathbf{t}_{i}$, and the conclusion follows.

Remark 5.3. Observe that the proofs of the last two lemmas go through in any normed linear space.

Theorem 5.4. The following statements hold:

(1) If $\mathbf{x}$ is an isolated extreme point of $B$, then there is a central projection $p \in N$ such that $\mathbf{x}=\Psi(p)$.

(2) If $N$ is finite-dimensional and $\mathbf{x}$ is an extreme point of $B$, then $\mathbf{x}=$ $\Psi(p)$ for some central projection $p \in N$, if and only if $\mathbf{x}$ is an isolated extreme point of $B$.

Proof. (1) Suppose $\mathbf{x}$ is an isolated extreme point in B. By Part (1) of Theorem 0.2 , there is a unique projection $p \in N$ such that $\mathbf{x}=\Psi(p)$. Next, applying Lemma 5.2 and Parts (2) and (3) of Theorem 0.2, we get linearly independent spectral pairs $\left(s_{1}, \mathbf{t}_{1}\right), \ldots,\left(s_{n+1}, \mathbf{t}_{n+1}\right)$ such that

$$
\{\mathbf{x}\}=\Psi\left(\left[p_{\mathbf{t}_{k}, s_{k}}^{-}, p_{\mathbf{t}_{k}, s_{k}}^{+}\right]\right), \quad k=1, \ldots, n+1 .
$$

Since $\Psi^{-1}(p)=\{\mathbf{x}\}$, we have

$$
p=p_{\mathbf{t}_{k}, s_{k}}^{ \pm} \quad k=1, \ldots, n+1 .
$$

Since these spectral pairs are linearly independent, a straightforward argument shows that the subspace of $\mathbb{R}^{n}$ spanned by $\mathbf{t}_{1}, \ldots, \mathbf{t}_{n+1}$ has dimension $n$. Hence, relabeling if necessary, we may assume that $\mathbf{t}_{1}, \ldots, \mathbf{t}_{n}$ are linearly independent. Therefore, by Corollary $4.7 p=p_{\mathbf{t}_{k}, s_{k}}^{ \pm}$is a central projection in $N$.

(2) Suppose that $\mathbf{x}=\Psi(p)$, where $p$ is a central projection in $N$ and let $\mathbf{x}_{m}$ denote a sequence of extreme points of $B$ that converges to $\mathbf{x}$. Applying Part (1) of Theorem 0.2 we get a sequence $p_{m}$ of unique projections in $N$ such that $\Psi\left(p_{m}\right)=\mathbf{x}_{m}$ for each $m$. Since $M$ is finite dimensional, we may pass to a subsequence and assume that the sequence $\left\{p_{m}\right\}$ is norm 
convergent with limit $q$. Since $\Psi$ is continuous, we have $\Psi(q)=\mathbf{x}=\Psi(p)$ and since $\Psi^{-1}(\mathbf{x})=\{p\}$, we get $q=p$. Now, since $p$ is central and the central projections are isolated in the set of all projections in $N$, we must have that $p_{m}=p$ (and hence $\mathbf{x}_{m}=\mathbf{x}$ ) for all sufficiently large $m$. Hence $\mathbf{x}$ is an isolated extreme point.

If $\mathbf{x}$ is an isolated extreme point in $B$, then by Part (1) of this Theorem, there is a central projection $p$ in $N$ such that $\mathbf{x}=\Psi(p)$.

\section{Remarks 5.5.}

(1) Let $b$ denote multiplication by $x$ on the interval $[0,1]$ and write $N=$ $\{b\}^{\prime \prime}=L^{\infty}(0,1)$. In this case every projection in $N$ is central but $B$ has no isolated extreme points. (See the example at the end of Section 1 in [1].) Hence, the converse to Part (1) of Theorem 5.4 is false.

(2) If $b, B$ and $N$ are as in Remark (1) above, then there are linearly independent spectral pairs $\left(s_{1}, \mathbf{t}_{1}\right)$ and $\left(s_{2}, \mathbf{t}_{2}\right)$ such that

$$
\{0\}=\Psi\left(\left[p_{\mathbf{t}_{i}, s_{i}}^{-}, p_{\mathbf{t}_{i}, s_{i}}^{+}\right]\right), \quad i=1,2,
$$

but 0 is not an isolated extreme point in $B$. (See [1].) Thus, the converse to Lemma 5.2 is also false.

Corollary 5.6. $N$ is abelian and has finite dimension if and only if $B$ has a finite number of extreme points.

Proof. If $N$ is abelian and has finite dimension, then each projection in $N$ is diagonal. Hence $N$ contains a finite number of projections and since every extreme point in $B$ is the image under $\Psi$ of a projection $N$, $\operatorname{ext}(B)$ is finite.

For the converse, if $B$ has a finite number of extreme points, then each extreme point in $B$ is an isolated extreme point and so by Part (1) of Theorem 5.4, each extreme point of $B$ has the form $\Psi(p)$, where $p$ is a central projection. On the other hand, if $(s, \mathbf{t})$ is any spectral pair, then $\Psi\left(p_{\mathbf{t}, s}^{ \pm}\right)$ is an extreme point by Part (1) of Theorem 0.2 and so $p_{\mathbf{t}, s}^{ \pm}$is a central projection. Thus, for each fixed $\mathbf{t}$ the von Neumann algebra

$$
\left\{p_{\mathbf{t}, s}^{ \pm}: s \in \mathbb{R}\right\}^{\prime \prime}
$$

is central and therefore each $b_{\mathbf{t}}$ is central. Hence $N$ is abelian. Since the projections of the form $p_{\mathbf{t}, s}^{ \pm}$generate $N$, this algebra has finite dimension.

\section{Countable extreme points.}

Our goal in this section is to establish the following Theorem.

Theorem 6.1. If the set $\operatorname{ext}(B)$ of extreme points of $B$ is countable, then $N$ is abelian.

It is convenient to present the bulk of the proof in a series of Lemmas. We begin with a simple topological fact. 
Lemma 6.2. If $X$ is a countable locally compact Hausdorff space, then the isolated points in $X$ are dense.

Proof. Suppose that the closure of the isolated points of $X$ is not all of $X$. By [5, Theorem 2.7] each open subset of $X$ is a locally compact Hausdorff space. Hence, there is a nonempty open set $U$ in $X$ with no isolated points. Since $X$ is countable, $U$ is countable and we may write $U=\left\{x_{1}, x_{2}, \ldots\right\}$. Set $U_{n}=U \backslash\left\{x_{n}\right\}$ for each $n$. As $X$ is Hausdorff, each $U_{n}$ is open and since $x_{n}$ is not isolated, each $U_{n}$ is dense in $U$. Hence by the Baire Category Theorem [4, Theorem $2.2(\mathrm{~b})], \cap U_{n}$ is dense in $U$. Since, $\cap U_{n}=\emptyset$, this is impossible. Hence, no such $U$ exists and so the isolated extreme points in $X$ are dense.

Lemma 6.3. It suffices to prove the theorem in the case where $n=2$.

Proof. Write $N_{i j}=\left\{b_{i}, b_{j}, 1\right\}^{\prime \prime}$, let $\pi_{i j}$ denote the map from $B$ to $\mathbb{R}^{3}$ defined by the formula

$$
\pi_{i j}\left(\left(\tau(a), \tau\left(b_{1} a\right), \ldots, \tau\left(b_{n} a\right)\right)\right)=\left(\tau(a), \tau\left(b_{i} a\right), \tau\left(b_{j} a\right)\right), \quad a \in N_{1}^{+}
$$

and write $B_{i j}=\pi_{i j}(B)$. It is clear that $B_{i j}=B\left(b_{i}, b_{j}\right)$.

Next suppose $\operatorname{ext}(B)$ is countable and fix $i$ and $j$. For each extreme point $\mathbf{x}$ in $B_{i, j}$ write $F_{\mathbf{x}}=\pi_{i j}^{-1}(\mathbf{x})$ so that $F_{\mathbf{x}}$ is a closed face of $B$. Now each $F_{\mathbf{x}}$ is nonempty and so contains extreme points. Since $F_{\mathbf{x}_{1}}$ and $F_{\mathbf{x}_{2}}$ are disjoint if $\mathbf{x}_{1}$ and $\mathbf{x}_{2}$ are distinct extreme points, the extreme points of $B_{i j}$ must be countable. Thus, if $\operatorname{ext}(B)$ is countable, then $\operatorname{ext}\left(B_{i j}\right)$ is countable for each pair $(i, j)$. Hence, if the Theorem is true when $n=2$, then each $N_{i j}$ is abelian and therefore $N$ is abelian.

For the remainder of this section, we shall assume that $N$ is the von Neumann algebra generated by $b_{1}, b_{2}$ and the identity and that $B=B\left(b_{1}, b_{2}\right)$ denotes the associated spectral scale. As $B \subset \mathbb{R}^{3}$ each face in $B$ must have dimension zero, one or two. Recall that for each real $s$, with $0 \leq s \leq 1$

$$
I_{s}=\{(s, y, z) \in B\}
$$

denotes the isotrace slice of $B$ at $s$. Since $I_{s}$ lives in a plane, we shall view it as a plane figure. In particular, when we refer to the boundary of $I_{s}$, we are considering it as a plane figure and the boundary is a curve.

Lemma 6.4. If $F$ is a face in $B$, then the following statements hold:

(1) If $F$ has dimension greater than zero, then $F$ is transverse to the isotrace slices of $B$.

(2) If $F$ has dimension one, then $F$ meets each isotrace slice in at most one point.

(3) If $F$ has dimension two, and $I_{s}$ contains a point in the relative interior of $F$, then $F \cap I_{s}$ is a proper line segment. 
Proof. Since $F$ is a face, there are projections $q^{-} \leq q^{+}$such that $F=$ $\Psi\left(\left[q^{-}, q^{+}\right]\right)$by Theorem 3.6. Now suppose that $F$ has dimension greater then zero. In this case we must have $q^{-}<q^{+}$so that $\tau\left(q^{-}\right)<\tau\left(q^{+}\right)$. Hence, $F$ is transverse to the isotrace slices of $B$ and so (1) is true.

The remaining assertions now follow easily from (1).

Lemma 6.5. If $0<s<1$ then the following statements hold for $I_{s}$.

(1) If $G$ is a proper closed line segment in the boundary of $I_{s}$, then there is a two dimensional face $F$ of $B$ such that $G \subset F \cap I_{s}$.

(2) If $\mathbf{x}$ is an extreme point of $I_{s}$, then either $\mathbf{x}$ is an extreme point of $B$ or else it lies in the relative interior of a one dimensional face of $B$.

(3) If $I_{s}$ is two dimensional and $\mathbf{x}$ is an extreme point of $I_{s}$ that is an isolated point in the set of extreme points of $I_{s}$, then there are two distinct two dimensional faces of $B$ that contain $\mathbf{x}$.

(4) If the set of extreme points of $B$ is countable then the set of extreme points of $I_{s}$ is countable.

Proof. Fix a closed proper line segment $G$ in the boundary of $I_{s}$. As $G$ is in the boundary of $I_{s}$, it is in the boundary of $B$ and so is contained in a minimal face $F$ in $B$. Since faces in $B$ of dimension greater than zero are transverse to the isotrace slices by Lemma 6.4(1), $F$ must be two dimensional. Hence (1) is true.

Next, fix an extreme point $\mathbf{x}$ of $I_{s}$. If $\mathbf{x}$ is not an extreme point of $B$, then it is properly contained in a minimal face $F$ of $B$. If $F$ were two dimensional, then by its minimality, we would get that $\mathbf{x}$ lies in the relative interior of $F$. But in this case $F \cap I_{s}$ would be a line segment by Lemma 6.4(2) and $\mathbf{x}$ would be in its interior, which is impossible since $\mathbf{x}$ is extreme in $I_{s}$. Hence, $F$ has dimension less than two. Since $\mathbf{x}$ is properly contained in $F$, this face must have dimension one. Finally, since $\mathbf{x}$ is not extreme in $B$, it must be contained in the relative interior of $F$ and so (2) is true.

Now suppose $\mathbf{x}$ is an isolated extreme point in $I_{s}$. Since $I_{s}$ has dimension 2 , its faces consist of extreme points and line segments. Since $\mathbf{x}$ is an isolated extreme point, it must be the endpoint of adjacent line segments in the boundary of $I_{s}$. Hence $\mathbf{x}$ lies in two distinct two dimensional faces of $B$ by Part (1) of this Lemma. Thus Part (3) is true.

Finally, assume that the set $\operatorname{ext}(B)$ of extreme points of $B$ is countable. Observe that each face in $B$ of dimension one is uniquely determined by its end points, which are extreme points in $B$ by Theorem 0.2 . Hence $B$ has a countable number of faces of dimension one.

Now fix $s$ with $0<s<1$. We have that $\operatorname{ext}(B) \cap I_{s}$ is countable. Each remaining extreme point of $I_{s}$ is contained in a unique face of $B$ with dimension one by Part (2) of this Lemma. Since there are a countable number of one dimensional faces in $B$, the set of extreme points of $I_{s}$ is countable. 
Lemma 6.6. If $\mathbf{x}$ is an isolated extreme point in $I_{s}$, then there are central projections $z_{1}$ and $z_{2}$ in $B$ and $0 \leq t \leq 1$ such that $z_{1} \leq z_{2}$ and

$$
\mathbf{x}=\Psi\left(t z_{1}+(1-t) z_{2}\right) .
$$

Proof. As $\mathrm{x}$ is isolated among the extreme points of $I_{s}$, there are distinct two dimensional faces $F_{1}$ and $F_{2}$ of $B$ such that $\mathbf{x} \in F_{1} \cap F_{2}=F$ by Part (3) of Lemma 6.5. Next, since $F_{1}$ and $F_{2}$ have maximal dimension in $B$, they must be exposed and so we may write

$$
F_{i}=\Psi\left(\left[p_{\mathbf{t}_{i}, s_{i}}^{-}, p_{\mathbf{t}_{i}, s_{i}}^{+}\right]\right), \quad i=1,2 .
$$

Further, since the $F_{i}$ 's intersect $I_{s}$ in adjoining line segments, we must have that $\mathbf{t}_{1} \neq \mathbf{t}_{2}$. Since neither of the $\mathbf{t}_{i}$ 's is zero, these vectors are linearly independent. Hence, by Corollary 4.7, we must have

$$
F=\Psi\left(\left[z_{1}, z_{2}\right]\right)
$$

where $z_{1}$ and $z_{2}$ are central projections in $N$. Finally, since the $F_{i}$ 's have dimension two, $F=F_{1} \cap F_{2}$ must be a (possibly degenerate) line segment. Hence elements of $F$ are the images under $\Psi$ of convex combinations of $z_{1}$ and $z_{2}$, as desired.

With these preparations, we may now present the proof of Theorem 6.1.

Proof. Write $\mathbf{t}_{1}=(1,0)$ and $\mathbf{t}_{2}=(0,1)$. To show that $N$ is abelian, it suffices to show that $p_{s, \mathbf{t}_{i}}^{ \pm}$is central for each spectral pair of the form $\left(s, \mathbf{t}_{i}\right), i=1,2$. Indeed in this case, by a standard argument in measure theory, we get that the spectral projections for $b_{1}$ and $b_{2}$ are central and so $b_{1}$ and $b_{2}$ commute.

So now let $p$ denote such a projection, write $\mathbf{x}=\Psi(p)$ and let $I_{s}$ denote the isotrace slice containing $\mathbf{x}$. By Part (1) of Theorem $0.2, \mathbf{x}$ is an extreme point of $B$ and so it is also an extreme point of $I_{s}$. Since $\operatorname{ext}(B)$ is countable, the extreme points of $I_{s}$ are countable by Part (4) of Lemma 6.5 and since $I_{s}$ has dimension 2 , these extreme points form a closed set. Hence, the isolated extreme points of $I_{s}$ are dense in $I_{s}$ by Lemma 6.2.

Now let $\left\{\mathbf{x}_{n}\right\}$ denote a sequence of isolated extreme point in $I_{s}$ that converges to $\mathbf{x}$. Applying Lemma 6.6 , we get sequences $\left\{z_{1, n}\right\},\left\{z_{2, n}\right\}$ of central projections in $N$ and a sequence $\left\{t_{n}\right\}$ of real numbers in $[0,1]$ such that

$$
\mathbf{x}_{n}=\Psi\left(t_{n} z_{1, n}+\left(1-t_{n}\right) z_{2, n}\right), \quad n=1,2, \ldots
$$

Write $a_{n}=t_{n} z_{1, n}+\left(1-t_{n}\right) z_{2, n}$ for each $n$. As the sequence $\left\{a_{n}\right\}$ lies in $N_{1}^{+}$, it is bounded and so it admits a subnet $\left\{a_{\alpha}\right\}$ that converges to an element 
$a$ in $N_{1}^{+}$in the weak*-topology. Since $\tau$ is normal, we have

$$
\begin{aligned}
\lim _{\alpha} \Psi\left(a_{\alpha}\right) & =\lim _{\alpha}\left(\tau\left(a_{\alpha}\right), \tau\left(b_{1} a_{\alpha}\right), \tau\left(b_{2} a_{\alpha}\right)\right) \\
& =\left(\tau(a), \tau\left(b_{1} a\right), \tau\left(b_{2} a\right)\right)=\Psi(a) \\
& =\mathbf{x}=\Psi(p) .
\end{aligned}
$$

Since $\Psi^{-1}(\mathbf{x})=\{p\}$ by Part (1) of Theorem 0.2, we get $a=p$. Since each $a_{n}$ is central and the center of $N$ is closed in the weak*-topology, we get that $p$ is central, as desired.

\section{References}

[1] C.A. Akemann, J. Anderson and N. Weaver, A geometric spectral theory for n-tuples of self-adjoint operators in finite von Neumann algebras, J. Functional Analysis, 164 (1999), 258-292, MR 2000f:46078, Zbl 0937.47003.

[2] J. Dixmier, $C^{*}$-Algebra, North-Holland, New York, 1977, MR 56 \#16388, Zbl 0372.46058 .

[3] R.T. Rockafellar, Convex Analysis, Princeton Univ. Press, Princeton, 1970, MR 43 \#445, Zbl 0193.18401.

[4] W. Rudin, Functional Analysis, $2^{\text {nd }}$ Edition, McGraw-Hill, New York, 1991, MR 92k:46001, Zbl 0867.46001.

[5] _ Real and Complex Analysis, McGraw-Hill, New York, 1987, MR 88k:00002, Zbl 0925.00005.

[6] R. Webster, Convexity, Oxford University Press, Oxford, 1994, MR 98h:52001, Zbl 0835.52001.

Received November 1, 2000. Each author was supported in part by The National Science Foundation during the preparation of this paper.

Department of Mathematics

UNIVERSITY OF CALIFORNIA

SANTA BARBARA, CA 93106

E-mail address: akemann@math.ucsb.edu

Department of Mathematics

Pennsylvania State University

UNIVERSITY PARK, PA 1680

E-mail address: anderson@math.psu.edu 